\title{
Two-loop off-shell QCD amplitudes in FDR
}

\section{Ben Page and Roberto Pittau}

CERN, PH-TH,

Geneva, Switzerland

Departamento de Física Teórica y del Cosmos and CAFPE, Universidad de Granada, Campus Fuentenueva s.n., E-18071 Granada, Spain

E-mail: ben.page@physik.uni-freiburg.de, pittau@ugr.es

ABSTRACT: We link the FDR treatment of ultraviolet (UV) divergences to dimensional regularization up to two loops in QCD. This allows us to derive the one-loop and two-loop coupling constant and quark mass shifts necessary to translate infrared finite quantities computed in FDR to the $\overline{\mathrm{MS}}$ renormalization scheme. As a by-product of our analysis, we solve a problem analogous to the historical difficulties of the Four Dimensional Helicity (FDH) method beyond one loop. An alternative formulation of FDH is then presented that preserves the renormalizability properties of $\mathrm{QCD}$ without introducing evanescent quantities.

KEYwords: Renormalization Regularization and Renormalons, QCD, Scattering Amplitudes

ARXIV EPRINT: 1506.09093 


\section{Contents}

1 Introduction 1

$\begin{array}{lll}2 & \text { FDR } & 3\end{array}$

$3 \quad$ FDR and DReg on the same footing 4

4 The calculation $\quad 5$

4.1 The one-loop case 6

$\begin{array}{lll}4.2 & \text { The two-loop case } & 7\end{array}$

4.3 "Extra"-extra integrals and sub-prescription 9

$\begin{array}{llr}5 & \text { Results } & 11\end{array}$

$\begin{array}{lll}6 & \text { FDH without evanescent quantities } & 13\end{array}$

$\begin{array}{lll}7 & \text { Summary and outlook } & 15\end{array}$

A The renormalization constants of the QCD vertices $\quad 16$

$\begin{array}{ll}\text { B A two-loop diagram } & 18\end{array}$

C Sub-prescription example $\quad 22$

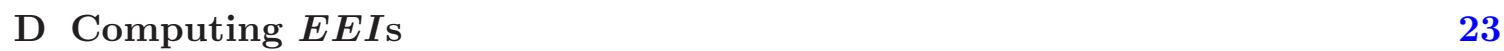

\section{Introduction}

When using customary approaches [1-5] to handle the ultraviolet (UV) problem in quantum field theory (QFT), intermediate steps are necessary to extract physical answers from loop calculations. In particular, the Lagrangian $\mathcal{L}$ of the theory is modified by adding UV counterterms (CTs). They absorb the divergences generated by the high-frequency part of the loop integrals and as one moves up the orders in the loop expansion one must include lower loop CT calculations in order to be consistent. When carrying out this renormalization program UV infinities are usually regulated via dimensional regularization [4] (DReg) and renormalized quantities are defined by specifying what is subtracted from the bare ones. A particularly convenient subtraction scheme is $\overline{\mathrm{MS}}$, in which only UV poles and universal constants are dropped.

The FDR ${ }^{1}$ approach of [6] deals with UV infinities in a different way. A new kind of loop integration ${ }^{2}$ is introduced that coincides with Riemann integration in UV finite cases,

\footnotetext{
${ }^{1}$ Acronym of Four Dimensional Regularization/Renormalization.

${ }^{2}$ Called FDR integration.
} 
but produces a finite and regulator free answer also when acting on divergent integrands. ${ }^{3}$ In this way no CTs need to be incorporated into $\mathcal{L}$ : they are traded for a change in the definition of the loop integration. Moreover, FDR directly generates renormalized amplitudes since it is independent of any UV cutoff.

The main aim of this paper is to construct the one- and two-loop transition rules between FDR and $\overline{\mathrm{MS}}$ in the framework of QCD. As a perturbative treatment of a renormalizable QFT is unique up to a renormalization scheme dependence, we have achieved this task by studying off-shell ${ }^{4} \ell$-loop QCD correlation functions $G^{\ell}$ and by searching for a DReg renormalization scheme which reproduces the FDR answer when $\ell=1$ and $\ell=2$, i.e.

$$
\begin{aligned}
& G_{\text {renormalized }}^{\ell}=G_{\text {bare }}^{\ell}+(\ell \text {-loop-CTs })+\ldots+(1 \text {-loop-CTs }), \\
& G_{\text {renormalized }}^{\ell}=G_{\mathrm{FDR}}^{\ell} .
\end{aligned}
$$

We dub such a scheme $\mathrm{D}_{\mathrm{Reg}}^{\mathrm{FDR}}$ and its renormalization constants $Z^{\ell, \mathrm{FDR}}$. These can then be used to extract the coupling constant and quark mass shifts that relate FDR to $\overline{\mathrm{MS}}$. We emphasize that in a typical FDR calculation there are no $Z^{\ell, F D R}$, since one directly computes eq. (1.1b). We introduce them in the context of this paper only because we want to work out the correspondence of FDR with a canonical renormalization approach based on counterterms.

The second insight of our work concerns the nature of self contractions of Lorentz indices and $\gamma$-matrices explicitly appearing in $G_{\mathrm{bare}}^{\ell}$, that we denote by $n_{s}$. When studying eq. (1.1) one needs to reconcile the value $n_{s}=n$ dictated by DReg in (1.1a) with $n_{s}=4$ used in (1.1b). This has to be done without spoiling the renormalizability of QCD. Following FDR as a guidance, we have been able to establish the correct $n_{s} \rightarrow 4$ limit of DReg, compatible with a local subtraction parametrized in terms of the $Z^{\ell, \mathrm{FDR}}$. A mismatch between the structure of $G_{\text {bare }}^{\ell}$ and the CTs has been recently recognized [7-9] to cause the failure of the renormalization program beyond one loop in the naive extension to non supersymmetric theories of the original formulation of the Four Dimensional Helicity scheme (FDH) [10, 11]. Since we observe that terms restoring the right cancellations are produced by FDR, we argue that the FDR approach provides a natural solution to this problem. In contrast with methods that require the introduction of $\epsilon$-scalars [12], in our case no new fields nor evanescent couplings need to be added to the QCD Lagrangian. Moreover, a shift linking the $\overline{\mathrm{MS}}$ quark mass to a fixed version of FDH can be worked out. So far we have tested this approach for off-shell correlation functions. What happens in the presence of IR divergences needs to be further investigated.

The outline of the paper is as follows. In the next section we review FDR. Sections 3 and 4 describe our computational strategy. Our results are collected in section 5 and appendix A. Furthermore, in section 6 we discuss our fix to FDH. Finally, appendices B-D contain explicit examples of our algorithm.

\footnotetext{
${ }^{3}$ Abusing a bit the language, we dub divergent(convergent) integrands those which would generate UV divergent(convergent) integrals upon normal, four-dimensional integration.

${ }^{4}$ Working off-shell allows us to deal with IR convergent integrals. A detailed two-loop study of FDR in the presence of IR divergences is equally important, but outside our focus.
} 


\section{FDR}

FDR integration was first introduced in [6] and several examples of one- and two-loop computations can be found in [13-17]. Here we briefly review its definition, which we need to make contact with our calculation.

Consider an $\ell$-loop integrand $J\left(q_{1}, \ldots, q_{\ell}\right)$ depending on $\ell$ loop momenta $q_{1}, \ldots, q_{\ell}$. The multi-loop FDR integration over $J$ is defined $\operatorname{as}^{5}$

$$
\int\left[d^{4} q_{1}\right] \cdots\left[d^{4} q_{\ell}\right] J\left(q_{1}, \ldots, q_{\ell}, \mu^{2}\right) \equiv \lim _{\mu \rightarrow 0} \int d^{4} q_{1} \cdots d^{4} q_{\ell} J_{\mathrm{F}}\left(q_{1}, \ldots, q_{\ell}, \mu^{2}\right),
$$

where $J_{\mathrm{F}}\left(q_{1}, \ldots, q_{\ell}, \mu^{2}\right)$ is the UV finite part of $J\left(q_{1}, \ldots, q_{\ell}, \mu^{2}\right)$, specified below, and $\mu$ a vanishing mass required to extract $J_{\mathrm{F}}$ from $J . J\left(q_{1}, \ldots, q_{\ell}, \mu^{2}\right)$ and $J_{\mathrm{F}}\left(q_{1}, \ldots, q_{\ell}, \mu^{2}\right)$ are read off from $J\left(q_{1}, \ldots, q_{\ell}\right)$ according to the following rules:

i) Squares of integration momenta appearing both in the denominators of $J\left(q_{1}, \ldots, q_{\ell}\right)$ and in contractions generated by Feynman rules are shifted by $\mu^{2}$

$$
q_{i}^{2} \rightarrow q_{i}^{2}-\mu^{2} \equiv \bar{q}_{i}^{2}
$$

This replacement is called global prescription;

ii) A splitting

$$
J\left(q_{1}, \ldots, q_{\ell}, \mu^{2}\right)=\left[J_{\mathrm{INF}}\left(q_{1}, \ldots, q_{\ell}, \mu^{2}\right)\right]+J_{\mathrm{F}}\left(q_{1}, \ldots, q_{\ell}, \mu^{2}\right)
$$

is performed in such a way that UV divergences are entirely parametrized in terms of divergent integrands ${ }^{6}$ contained in $\left[J_{\mathrm{INF}}\right]$, that solely depend on $\mu^{2}$;

iii) The global prescription of eq. (2.2) should be made compatible with a key property of multi-loop calculus: ${ }^{7}$

in an $\ell$-loop diagram, one should be able to calculate a sub-diagram, insert the integrated form into the full diagram and get the same answer.

We dub this sub-integration consistency.

Finally, after $\lim _{\mu \rightarrow 0}$ is taken, $\ln \mu \rightarrow \ln \mu_{\mathrm{R}}$ is understood in the r.h.s. of eq. (2.1), where $\mu_{\mathrm{R}}$ is an arbitrary renormalization scale.

This definition preserves shift invariance

$$
\int\left[d^{4} q_{1}\right] \ldots\left[d^{4} q_{\ell}\right] J\left(q_{1}, \ldots, q_{\ell}, \mu^{2}\right)=\int\left[d^{4} q_{1}\right] \ldots\left[d^{4} q_{\ell}\right] J\left(q_{1}+p_{1}, \ldots, q_{\ell}+p_{\ell}, \mu^{2}\right),
$$

\footnotetext{
${ }^{5} \mathrm{FDR}$ integration is denoted by the symbol $\left[d^{4} q_{i}\right]$.

${ }^{6}$ By convention, we write divergent integrands between square brackets and call them FDR vacua, or simply vacua. Examples of the extraction of FDR vacua from loop integrands are given in appendix B.

${ }^{7}$ This last requirement turns out to be the mechanism that enforces the renormalizability of the $\mathrm{D}_{\text {Reg }}^{\mathrm{FDR}}$ scheme. It is discussed at length in section 4.3 .
} 
and the possibility of cancelling numerators and denominators

$$
\int\left[d^{4} q_{1}\right] \ldots\left[d^{4} q_{\ell}\right] \frac{\bar{q}_{i}^{2}-m_{i}^{2}}{\left(\bar{q}_{i}^{2}-m_{i}^{2}\right)^{m} \ldots}=\int\left[d^{4} q_{1}\right] \ldots\left[d^{4} q_{\ell}\right] \frac{1}{\left(\bar{q}_{i}^{2}-m_{i}^{2}\right)^{m-1} \ldots},
$$

which are properties needed to retain the symmetries of $\mathcal{L}$. From eqs. $(2.5)$ and $(2.6)$ it follows that algebraic manipulations in FDR integrands are allowed as if they where convergent ones. This authorizes one to reduce complicated multi-loop integrals to a limited set of Master Integrals (MI) by using four-dimensional tensor decomposition [14] or integrationby-parts identities [17]. In other words, the definition in eq. (2.1) can be applied just at the end of the calculation, when the actual value of the MIs is needed.

An important subtlety implied by eq. (2.6) is that the needed cancellation works only if integrands involving explicit powers of $\mu^{2}$ in the numerator are also split via eq. (2.3) as if $\mu^{2}=q_{i}^{2}$, where $q_{i}^{2}$ is the momentum squared which generates $\mu^{2}$. As a consequence, although only one kind of $\mu^{2}$ exists, one has to keep track of its origin when it appears in the numerator of $J\left(q_{1}, \ldots, q_{\ell}, \mu^{2}\right)$. For this we use the notation $\left.\mu^{2}\right|_{i}$, which understands the same splitting required for $q_{i}^{2}$. FDR integrals with powers of $\left.\mu^{2}\right|_{i}$ in the numerator are called "extra integrals". Their computation is elementary. One- and two-loop examples can be found in $[6,14]$ and appendix D.

\section{FDR and DReg on the same footing}

Eq. (1.1) defines the $\mathrm{D}_{\mathrm{Reg}}^{\mathrm{FDR}}$ scheme we are looking for, i.e. the DReg scheme reproducing the FDR correlation functions. As the r.h.s. of (1.1a) is computed in DReg while the r.h.s. of (1.1b) in FDR, we need a common framework which accommodates both approaches. Here we illustrate this framework at two loops, but the same considerations apply at any loop order.

Our starting point is eq. (2.3) when $J\left(q_{1}, q_{2}\right)$ is the sum of all integrands contributing

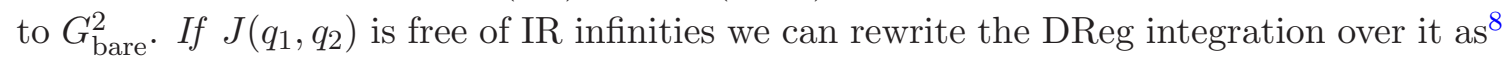

$$
\begin{aligned}
& \int d^{n} q_{1} d^{n} q_{2} J\left(q_{1}, q_{2}\right)=\int d^{n} q_{1} d^{n} q_{2} \lim _{\mu \rightarrow 0} J\left(q_{1}, q_{2}, \mu^{2}\right)=\lim _{\mu \rightarrow 0} \int d^{n} q_{1} d^{n} q_{2} J\left(q_{1}, q_{2}, \mu^{2}\right) \\
& =\lim _{\mu \rightarrow 0} \int d^{n} q_{1} d^{n} q_{2}\left[J_{\mathrm{INF}}\left(q_{1}, q_{2}, \mu^{2}\right)\right]+\lim _{\mu \rightarrow 0} \int d^{4} q_{1} d^{4} q_{2} J_{\mathrm{F}}\left(q_{1}, q_{2}, \mu^{2}\right)
\end{aligned}
$$

where the absence of IR divergences authorizes us to extract $\lim _{\mu \rightarrow 0}$ out of the integral. The integral over $J_{\mathrm{F}}\left(q_{1}, q_{2}, \mu^{2}\right)$ is $G_{\mathrm{FDR}}^{2}$ and through eq. (3.1) we have isolated it within $G_{\text {bare }}^{2}$, so that the $\mathrm{D}_{\mathrm{Reg}}^{\mathrm{FDR}}$ scheme can be determined by solely looking at the pieces which differ between eq. (1.1a) and (1.1b), namely

$$
\left[J_{\mathrm{INF}}\left(q_{1}, q_{2}, \mu^{2}\right)\right], \quad(1 \text {-loop-CTs) and (2-loop-CTs). }
$$

This defines our strategy. For instance the (1-loop-CTs) are known at two loops, so that $\mathrm{D}_{\mathrm{Reg}}^{\mathrm{FDR}}$ is defined by choosing

$$
(2 \text {-loop-CTs })=-(1 \text {-loop-CTs })-\lim _{\mu \rightarrow 0} \int d^{n} q_{1} d^{n} q_{2}\left[J_{\mathrm{INF}}\left(q_{1}, q_{2}, \mu^{2}\right)\right],
$$

\footnotetext{
${ }^{8} n$ is the number of dimensions defined as $n=4-2 \epsilon$.
} 
which sets the finite part of $G_{\text {bare }}^{2}$ to the FDR value.

An important condition should be fulfilled by eq. (3.3). It should be local and proportional to the Born correlation function $G^{0}$ :

$$
(2 \text {-loop-CTs })=\text { Const } \times G^{0},
$$

which is the correct form to be re-absorbed into $Z^{2 \text { FDR }}$. We dub eq. (3.4) our renormalizability condition. To understand its consequences it is convenient to split $\left[J_{\mathrm{INF}}\right]$ as follows:

$$
\left[J_{\mathrm{INF}}\left(q_{1}, q_{2}, \mu^{2}\right)\right]=\left[J_{\mathrm{INF}}^{\mathrm{SV}}\left(q_{1}, q_{2}, \mu^{2}\right)\right]+\left[J_{\mathrm{INF}}^{\mathrm{GV}}\left(q_{1}, q_{2}, \mu^{2}\right)\right] .
$$

$\left[J_{\mathrm{INF}}^{\mathrm{SV}}\right]$ is a factorizable contribution in which only one sub-integration is UV divergent. It is called the sub-vacuum and depends on the kinematic scales entering the UV finite sub-integration. The second piece is independent of kinematics and it is called the global vacuum..$^{9}$ Eqs. (3.3) and (3.4) require that, upon integration, kinematic dependent terms in $\left[J_{\mathrm{INF}}^{\mathrm{SV}}\right]$ should cancel one-loop counterterms. In section 4 we will describe the subtleties of this cancellation.

\section{The calculation}

As outlined in the previous section the core of our strategy is calculating FDR vacua in DReg instead of throwing them away. As a first step we write down ${ }^{10}$ the twelve integrands $J^{G_{i}^{\ell}}(i=1, \ldots, 6$ and $\ell=1,2)$ corresponding to the one particle irreducible one- and twoloop QCD correlation functions drawn in figure 1, excluding CT diagrams. Then, we split them as in eq. (2.3) and compute the integrals over their vacuum parts,

$$
I_{i}^{1}=\lim _{\mu \rightarrow 0} \int \frac{d^{n} q}{\mu_{\mathrm{R}}^{-2 \epsilon}}\left[J_{\mathrm{INF}}^{G_{i}^{1}}\left(q, \mu^{2}\right)\right] \quad \text { and } \quad I_{i}^{2}=\lim _{\mu \rightarrow 0} \int \frac{d^{n} q_{1}}{\mu_{\mathrm{R}}^{-2 \epsilon}} \frac{d^{n} q_{2}}{\mu_{\mathrm{R}}^{-2 \epsilon}}\left[J_{\mathrm{INF}}^{G_{i}^{2}}\left(q_{1}, q_{2}, \mu^{2}\right)\right],
$$

in the symmetric off-shell kinematic point

$$
k_{1}^{2}=k_{2}^{2}=k_{3}^{2}=M^{2} .
$$

With this choice all integrals are made free of IR divergences, so eq. (3.1) applies. Furthermore, the complexity of the calculation is reduced to a one scale problem.

An important point concerns explicit contractions appearing in the numerator of $\left[J_{\mathrm{INF}}^{G_{i}^{\ell}}\left(q, \mu^{2}\right)\right]$ :

$$
\gamma^{\alpha} \gamma_{\alpha}=g^{\alpha \beta} g_{\alpha \beta}=n_{s}
$$

As $I_{i}^{1}$ and $I_{i}^{2}$ are regulated in DReg, $n_{s}=n$ should be used in eq. (4.1).

The knowledge of the $I_{i}^{\ell}$ allows us to parametrize the FDR subtraction in terms of renormalization constants $Z_{i}^{\ell, F D R}$. However, not all of them are independent, as they are related by QCD Slavnov-Taylor (S-T) identities. ${ }^{11}$ In the following we consider, in turn, the $\ell=1$ and $\ell=2$ contributions to the $Z_{i}^{\ell, \mathrm{FDR}}$.

\footnotetext{
${ }^{9}$ Examples of global vacua and sub-vacua are given in the first line of eq. (B.14) and in eq. (B.17), respectively.

${ }^{10}$ We work in the Feynman-'t Hooft gauge using QGRAF [18] for generating the diagrams and FORM [19] for extracting their vacuum part.

${ }^{11}$ See section 5 .
} 

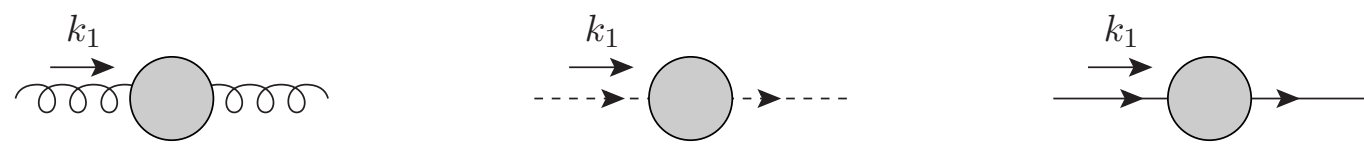

$$
G_{1}^{\ell}=G_{G G}^{\ell}
$$

$$
G_{2}^{\ell}=G_{c c}^{\ell}
$$

$$
G_{3}^{\ell}=G_{\Psi \Psi}^{\ell}
$$
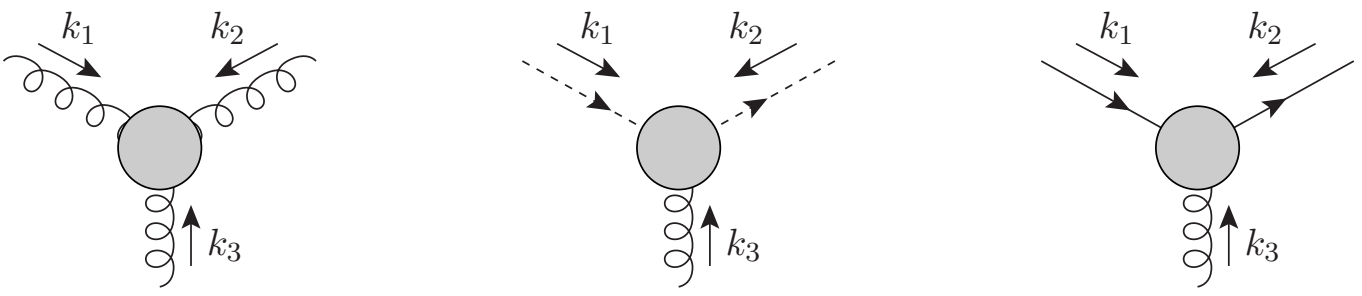

$G_{4}^{\ell}=G_{G G G}^{\ell}$

$$
G_{5}^{\ell}=G_{G c c}^{\ell}
$$

$$
G_{6}^{\ell}=G_{G \Psi \Psi}^{\ell}
$$

Figure 1. One particle irreducible one-loop $(\ell=1)$ and two-loop $(\ell=2)$ QCD Green's functions used in our calculation. The gray blobs denote the sum of all possible Feynman diagrams computed in the Feynman-'t Hooft gauge. Diagrams with counterterms are not included when the $G_{i}^{\ell}$ are used to compute the r.h.s. of eq. (1.1b).

\subsection{The one-loop case}

At one loop the calculation of $I_{i}^{1}$ is simple. Schematically:

- The global prescription of eq. (2.2) ensures that reducible $\bar{q}^{2} \mathrm{~s}$ in the numerator simplify with denominators;

- The integrands are then split as in eq. (2.3) by using the propagator identity

$$
\frac{1}{\bar{q}^{2}+2(q \cdot k)+k^{2}}=\frac{1}{\bar{q}^{2}}-\frac{2(q \cdot k)+k^{2}}{\bar{q}^{2}\left(\bar{q}^{2}+2(q \cdot k)+k^{2}\right)},
$$

which allows one to express $I_{i}^{1}$ in terms of tensors of the kind

$$
\int \frac{d^{n} q}{\mu_{\mathrm{R}}^{-2 \epsilon}} \frac{q^{\alpha_{1}} \cdots q^{\alpha_{2 r}}}{\left(q^{2}-\mu^{2}\right)^{s}}, \quad(4+2 r-2 s \geq 0)
$$

- Finally, by virtue of $\lim _{\mu \rightarrow 0}$, polynomially divergent integrals vanish, so any tensor structure is reduced to a fundamental scalar

$$
\int \frac{d^{n} q}{\mu_{\mathrm{R}}^{-2 \epsilon}} \frac{q^{\alpha_{1}} \cdots q^{\alpha_{2 r}}}{\left(q^{2}-\mu^{2}\right)^{r+2}}=\frac{2}{(2 r+2) ! !} g^{\alpha_{1} \cdots \alpha_{2 r}} V(\mu),
$$

with

$$
\begin{aligned}
V(\mu) & \equiv \int \frac{d^{n} q}{\mu_{\mathrm{R}}^{-2 \epsilon}} \frac{1}{\left(q^{2}-\mu^{2}\right)^{2}}=i \pi^{2}\left(\Delta-\ln \frac{\mu^{2}}{\mu_{\mathrm{R}}^{2}}\right)+\mathcal{O}(\epsilon), \\
\Delta & =\frac{1}{\epsilon}-\gamma_{E}-\ln \pi,
\end{aligned}
$$

and where $g^{\alpha_{1} \cdots \alpha_{2 r}}$ is completely symmetric and only made of products of metric tensors. 
Eventually, the logarithm in eq. (4.7) has to be combined with the one-loop analogue of the $J_{\mathrm{F}}$ term in eq. (3.1) to compensate the $\mu$ dependence of the finite part. ${ }^{12}$ As a result, the UV divergent part of $I_{i}^{1}, \operatorname{Inf}\left(I_{i}^{1}\right)$, is fully proportional to ${ }^{13}$

$$
V_{0}=\left.V\left(\mu_{\mathrm{R}}\right)\right|_{\epsilon \rightarrow 0}=i \pi^{2} \Delta .
$$

Now we study $\operatorname{Fin}\left(I_{i}^{1}\right)$, namely the UV convergent contribution to $I_{i}^{1}$. It determines the finite part of the one-loop subtraction term in eq. (1.1a) and appears due to the explicit presence of $n_{s}$ in $\left[J_{\mathrm{INF}}^{G_{i}^{1}}\left(q, \mu^{2}\right)\right]$. In fact, rewriting

$$
n_{s}=4-2 \lambda \epsilon,
$$

we see that when $\lambda \epsilon$ multiplies UV single poles, constants are generated that are fully subtracted in $\mathrm{D}_{\mathrm{Reg}}^{\mathrm{FDR}}$ but contribute to the finite part of $G_{i}^{1}$ in $\overline{\mathrm{MS}}$. Thus, the requirement of eq. (1.1b) causes a deviation from the minimality of the $Z_{i}^{1, \text { FDR }}$ proportional to $(1-\lambda)$, such that $\lambda=0$ (1) in $\mathrm{D}_{\mathrm{Reg}}^{\mathrm{FDR}}(\overline{\mathrm{MS}})$. As one finds that both $\operatorname{Inf}\left(I_{i}^{1}\right)$ and $\operatorname{Fin}\left(I_{i}^{1}\right)$ factorize the Born, one computes

$$
Z_{i}^{1, \mathrm{FDR}} G_{i}^{0}=-I_{i}^{1}
$$

In summary, at one loop it is possible to perform a calculation in DReg and consistently renormalize the result to reproduce the FDR answer. In other words, FDR can be interpreted as a particular renormalization scheme of DReg, i.e. the $\mathrm{D}_{\mathrm{Reg}}^{\mathrm{FDR}}$ scheme we are looking for.

\subsection{The two-loop case}

Here we illustrate the calculation for massless QCD. When dealing with a non-vanishing quark mass $m_{q}$ the formulae complicate a bit, but the reasoning remains unchanged. For simplicity, we start from cases ${ }^{14}$ in which we see that the sub-integration consistency (2.4) does not play any role, and postpone to section 4.3 the study of more complicated situations.

After scalarization by means of tensor reduction and integration-by-parts, one finds two types of contributions to $I_{i}^{2}$ :

- An integral over the global vacuum of the kind

$$
G V_{i}(\mu)=\lim _{\mu \rightarrow 0} \int \frac{d^{n} q_{1}}{\mu_{\mathrm{R}}^{-2 \epsilon}} \frac{d^{n} q_{2}}{\mu_{\mathrm{R}}^{-2 \epsilon}}\left(F_{i 1}\left(n, n_{s}\right)\left[\frac{1}{\bar{q}_{1}^{4} \bar{q}_{2}^{4}}\right]+F_{i 2}\left(n, n_{s}\right)\left[\frac{1}{\bar{q}_{1}^{4} \bar{q}_{2}^{2} \bar{q}_{12}^{2}}\right]\right),
$$

where $F_{i j}\left(n, n_{s}\right)$ are rational functions and $q_{12}=q_{1}+q_{2}$;

- An integral over the factorizable sub-vacuum ${ }^{15}$ of the form

$$
S V_{i}(\mu)=\lim _{\mu \rightarrow 0} \int \frac{d^{n} q_{1}}{\mu_{\mathrm{R}}^{-2 \epsilon}} \frac{d^{n} q_{2}}{\mu_{\mathrm{R}}^{-2 \epsilon}}\left[\frac{1}{\bar{q}_{2}^{4}}\right] J_{i}\left(q_{1}, \mu\right),
$$

in which $J_{i}\left(q_{1}, \mu\right)$ is UV convergent.

\footnotetext{
${ }^{12}$ This is why FDR integration is defined with the replacement $\ln \mu \rightarrow \ln \mu_{\mathrm{R}}$.

${ }^{13}$ The notation $\left.\right|_{\epsilon \rightarrow 0}$ means neglecting terms of $\mathcal{O}(\epsilon)$.

${ }^{14}$ Specified later on.

${ }^{15}$ This sub-vacuum is generated by the application of the identities (with $i \neq j$ )

$\frac{1}{\bar{q}_{i}^{2}}=\frac{1}{\bar{q}_{j}^{2}}\left(1-\frac{q_{i j}^{2}-2\left(q_{j} \cdot q_{i j}\right)}{\bar{q}_{i}^{2}}\right), \frac{1}{\bar{q}_{i}^{2}}=\frac{1}{\bar{q}_{i j}^{2}}\left(1+\frac{q_{j}^{2}+2\left(q_{i} \cdot q_{j}\right)}{\bar{q}_{i}^{2}}\right), \frac{1}{\bar{q}_{i j}^{2}}=\frac{1}{\bar{q}_{i}^{2}}\left(1-\frac{q_{j}^{2}+2\left(q_{i} \cdot q_{j}\right)}{\bar{q}_{i j}^{2}}\right)$
}

needed to disentangle the sub-divergences of $I_{i}^{2}$ from the finite part. 
By judiciously using the identity

$$
\frac{1}{\bar{q}_{1}^{2}+2\left(q_{1} \cdot k\right)+k^{2}}=\frac{1}{k^{2}}-\frac{\bar{q}_{1}^{2}+2\left(q_{1} \cdot k\right)}{k^{2}\left(\bar{q}_{1}^{2}+2\left(q_{1} \cdot k\right)+k^{2}\right)}
$$

$J_{i}\left(q_{1}, \mu\right)$ can be split into a piece which develops a $\ln \mu^{2}$ upon integration and a term where $\mu$ can be set to zero at the integrand level

$$
J_{i}\left(q_{1}, \mu\right)=J_{a i}\left(q_{1}, \mu\right)+J_{b i}\left(q_{1}\right) .
$$

With our special kinematics we find

$$
\begin{aligned}
J_{a i}\left(q_{1}, \mu\right) & =\frac{A_{i}\left(n, n_{s}\right)}{\bar{q}_{1}^{4}}, \\
J_{b i}\left(q_{1}\right) & =\frac{A_{i}^{\prime}\left(n, n_{s}\right)}{q_{1}^{2}\left(q_{1}+k_{1}\right)^{2}}+M^{2} \frac{B_{i}\left(n, n_{s}\right)}{q_{1}^{2}\left(q_{1}-k_{1}\right)^{2}\left(q_{1}+k_{2}\right)^{2}},
\end{aligned}
$$

where $A_{i}\left(n, n_{s}\right), A_{i}^{\prime}\left(n, n_{s}\right)$ and $B_{i}\left(n, n_{s}\right)$ are rational functions. Notice that the UV finiteness of $J_{i}\left(q_{1}, \mu\right)$ ensures that the pole parts of the integrals over $J_{a i}\left(q_{1}, \mu\right)$ and $J_{b i}\left(q_{1}\right)$ cancel each other. Furthermore, $B_{i}\left(n, n_{s}\right)=0$ in the case of two-point correlation functions. An explicit example of the procedure yielding eqs. (4.12)-(4.17) is reported in appendix B.

The additional complexity at two loops is that, unlike

$$
S V_{a i}(\mu)=\lim _{\mu \rightarrow 0} \int \frac{d^{n} q_{1}}{\mu_{\mathrm{R}}^{-2 \epsilon}} \frac{d^{n} q_{2}}{\mu_{\mathrm{R}}^{-2 \epsilon}}\left[\frac{1}{\bar{q}_{2}^{4}}\right] J_{a i}\left(q_{1}, \mu\right),
$$

the integral

$$
S V_{b i}=\lim _{\mu \rightarrow 0} \int \frac{d^{n} q_{1}}{\mu_{\mathrm{R}}^{-2 \epsilon}} \frac{d^{n} q_{2}}{\mu_{\mathrm{R}}^{-2 \epsilon}}\left[\frac{1}{\bar{q}_{2}^{4}}\right] J_{b i}\left(q_{1}\right)
$$

depends on the kinematics. Thus, it generates logarithms of physical scales that cannot be absorbed into the $Z_{i}^{2, F D R}$. Therefore eq. (3.3) tells us that, in order to establish the connection between FDR and the standard renormalization approach, we must demonstrate that such non-local terms are compensated by the sum of all diagrams containing insertions of one-loop counterterms. ${ }^{16}$ This contribution is dubbed $C T_{i}$ in the following. To achieve this cancellation, we have to recast eq. (4.19) into a form suitable to be combined with the $C T_{i}$. Observing that ${ }^{17}$

$$
S V_{i}(\mu)=V_{0} \lim _{\mu \rightarrow 0} \int \frac{d^{n} q_{1}}{\mu_{\mathrm{R}}^{-2 \epsilon}} J_{i}\left(q_{1}, \mu\right)+\mathcal{O}(\epsilon)
$$

leads us to consider

$$
\begin{aligned}
S V_{a i}^{\prime}(\mu) & =V_{0} \lim _{\mu \rightarrow 0} \int \frac{d^{n} q_{1}}{\mu_{\mathrm{R}}^{-2 \epsilon}} J_{a i}\left(q_{1}, \mu\right), \\
S V_{b i}^{\prime} & =V_{0} \lim _{\mu \rightarrow 0} \int \frac{d^{n} q_{1}}{\mu_{\mathrm{R}}^{-2 \epsilon}} J_{b i}\left(q_{1}\right)
\end{aligned}
$$

\footnotetext{
${ }^{16}$ For consistency with the form of the $Z_{i}^{1, F D R}$ the one-loop insertions have to be computed with $\lambda=0$.

${ }^{17} V_{0}$ is defined in eq. (4.9).
} 
instead of eqs. (4.18) and (4.19). Now $S V_{b i}^{\prime}$ has the same structure of a counterterm diagram, as it factorizes $V_{0}$. An explicit calculation of the $C T_{i}$ gives

$$
C T_{i}+S V_{b i}^{\prime}=0 \text { for } i=1,2,4,5,
$$

and we obtain, for correlation functions without external fermions, the following two-loop renormalization constants

$$
Z_{i}^{2, \mathrm{FDR}} G_{i}^{0}=-G V_{i}\left(\mu_{\mathrm{R}}\right)-S V_{a i}^{\prime}\left(\mu_{\mathrm{R}}\right),
$$

in accordance with the renormalizability condition of eq. (3.4). As in eq. (4.9), the choice of the point $\mu=\mu_{\mathrm{R}}$ has the effect of removing the dependence on $\mu$ from the last integral in eq. (3.1).

As in the one-loop case, $n_{s}$ in eqs. (4.12) and (4.17) generates finite terms proportional to $(1-\lambda)$ in the $Z_{i}^{2, F D R}$. In addition, finite contributions are created by two-loop integrands that do not multiply powers of $\lambda \epsilon$, e.g.

$$
\left[\frac{f(n)}{\bar{q}_{1}^{4} \bar{q}_{2}^{2} \bar{q}_{12}^{2}}\right]
$$

To denote their origin we multiply by a parameter $\delta$ any finite combination

$$
\int d^{n} q_{1} d^{n} q_{2}\left[\frac{f(n)}{\bar{q}_{1}^{4} \bar{q}_{2}^{2} \bar{q}_{12}^{2}}\right]-\text { Pole Part }\left\{\int d^{n} q_{1} d^{n} q_{2}\left[\frac{f(n)}{\bar{q}_{1}^{4} \bar{q}_{2}^{2} \bar{q}_{12}^{2}}\right]\right\}
$$

which is fully subtracted in $\mathrm{D}_{\mathrm{Reg}}^{\mathrm{FDR}}$ but contributes to the finite part of $G_{i}^{2}$ in $\overline{\mathrm{MS}}$, so that $\delta=1(0)$ in $\mathrm{D}_{\mathrm{Reg}}^{\mathrm{FDR}}(\overline{\mathrm{MS}})$.

\section{3 "Extra"-extra integrals and sub-prescription}

In the case of QCD Green's functions with external fermions eq. (4.22) does not hold true ${ }^{18}$ and leads to results incompatible with eq. (3.4). In this section, we show that the FDR subintegration consistency (2.4) requires the introduction in the finite part of the correlation functions of "extra"-extra integrals $(E E I \mathrm{~s})$ with the same structure of $S V_{i}^{\prime}$, namely

$$
E E I_{i}(\mu)=E E I_{a i}(\mu)+E E I_{b i}
$$

They are just what is needed to restore the renormalizability condition, i.e. ${ }^{19}$

$$
C T_{i}+S V_{b i}^{\prime}-E E I_{a i}(\mu)-E E I_{b i}=-E E I_{a i}(\mu)
$$

and

$$
Z_{i}^{2, \mathrm{FDR}} G_{i}^{0}=-G V_{i}\left(\mu_{\mathrm{R}}\right)-S V_{a i}^{\prime}\left(\mu_{\mathrm{R}}\right)+E E I_{a i}\left(\mu_{\mathrm{R}}\right) \quad(i=3,6),
$$

with $E E I_{a i}$ independent of physical scales.

\footnotetext{
${ }^{18}$ Interestingly, it applies when computing $I_{3}^{2}$ and $I_{6}^{2}$ with $n_{s}=4$, which is the value prescribed by FDH, as discusses in section 6 . Nevertheless, we emphasize that we must set $n_{s}$ to $n$ because our strategy is to reproduce the FDR result by regularizing FDR vacua in DReg, which dictates $n_{s}=n$.

${ }^{19}$ When calculating the vacuum $E E I$ s contribute with a minus sign and should be computed with $n_{s}=n$.
} 


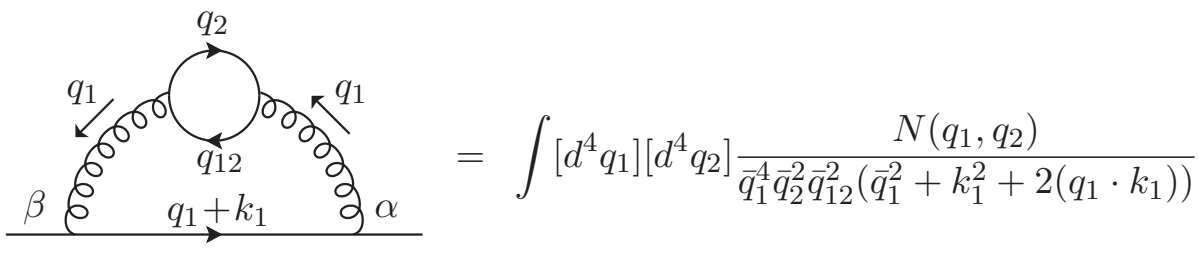

Figure 2. The two-loop diagram contributing the $N_{f}$ corrections to $G_{3}^{2} . N\left(q_{1}, q_{2}\right)$ is given in the text dropping irrelevant constants. The replacement $q_{i}^{2} \rightarrow \bar{q}_{i}^{2}$ is performed only in the denominators.

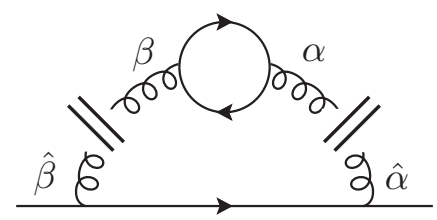

Figure 3. The same diagram of figure 2 from the point of view of the $q_{2}$ sub-integration. Lorentz indices external to the sub-diagram are given a hat.

The FDR origin of the $E E I_{i}$ is the need of introducing a sub-prescription to cure the mismatch between the global prescription of eq. (2.2) and the consistency condition (2.4). In fact, although essential to preserve gauge invariance at two loops, the shift

$$
q_{i}^{2} \rightarrow q_{i}^{2}-\left.\mu^{2}\right|_{i}
$$

may clash with the analogous replacement required to ensure (2.4) at the level of divergent one-loop sub-diagrams. This is better explained with an example. Consider the two-loop $\operatorname{diagram}^{20}$ of figure 2. Before applying eq. (4.29) its numerator reads

$$
N\left(q_{1}, q_{2}\right)=4 \gamma_{\alpha}\left(\not q_{1}+\not k_{1}\right) \gamma_{\beta}\left(-g^{\alpha \beta}\left(q_{2} \cdot q_{12}\right)+q_{12}^{\alpha} q_{2}^{\beta}+q_{12}^{\beta} q_{2}^{\alpha}\right) .
$$

The sub-prescription is defined as the effect of eq. (4.29) on $N\left(q_{1}, q_{2}\right)$ from the point of view of the divergent sub-diagram pulled out from the rest of the diagram, as in figure 3. From the perspective of the $q_{2}$ sub-integration one has to distinguish internal and external parts. We denote the separation of the parts "external" to the sub-diagram by placing hats on the Lorentz indices. Algebraically the hats do not make any difference other than to denote the fact that they are of an origin which is external to the sub-diagram, so all standard identities apply, for example

$$
\gamma_{\hat{\alpha}} \gamma^{\hat{\alpha}}=\gamma_{\alpha} \gamma^{\hat{\alpha}}=4, \quad \gamma_{\hat{\alpha}} q_{2}{ }^{\alpha}=\not_{2} .
$$

We then study how the part ${ }^{21}$ of $N\left(q_{1}, q_{2}\right)$ divergent in $q_{2}$

$$
N^{(2)}\left(q_{1}, q_{2}\right)=8\left(\not q_{1}+\not k_{1}\right) q_{2}^{2}+8 \not q_{2}\left(\not q_{1}+\not k_{1}\right) \not \not_{2},
$$

\footnotetext{
${ }^{20}$ This is the only possible contribution to $G_{3}^{2}$ proportional to $N_{f}$.

${ }^{21}$ Here this is quadratic because less-than-quadratic terms do not generate extra integrals.
} 
transforms under eq. (4.29). From the perspective of the sub-prescription the second term is inert, since it has an origin external to the sub-diagram. Thus

$$
N^{(2)}\left(q_{1}, q_{2}\right) \rightarrow N^{(2)}\left(q_{1}, q_{2}\right)-\left.8\left(\not q_{1}+\not k_{1}\right) \mu^{2}\right|_{2} .
$$

On the other hand, the two-loop global prescription dictates that also the second term must transform as ${ }^{22}$

$$
8 \not_{2}\left(\not \not_{1}+\not k_{1}\right) \not_{2} \rightarrow 8 \not_{2}\left(\not_{1}+\not k_{1}\right) \not_{2}+\left.8\left(\not \not_{1}+\not k_{1}\right) \mu^{2}\right|_{2},
$$

leading to no change in $N^{(2)}$ :

$$
N^{(2)}\left(q_{1}, q_{2}\right) \rightarrow N^{(2)}\left(q_{1}, q_{2}\right)
$$

Therefore, to remove double counting, the $E E I$ to be added to the diagram in figure 2 is defined as the result of the sub-prescription minus the outcome of the global prescription, i.e. the difference ${ }^{23}$ between the r.h.s. of eqs. (4.33) and (4.35)

$$
E E I=-8 \int\left[d^{4} q_{1}\right]\left[d^{4} q_{2}\right] \frac{\left.\hat{\mu}^{2}\right|_{2}\left(\not \phi_{1}+\not k_{1}\right)}{\bar{q}_{1}^{4} \bar{q}_{2}^{2} \bar{q}_{12}^{2}\left(\bar{q}_{1}^{2}+k_{1}^{2}+2\left(q_{1} \cdot k_{1}\right)\right)} .
$$

Here, we mark the $\left.\hat{\mu}^{2}\right|_{2}$ with a hat because it is acting only on the $q_{2}$ sub-integral. One finds ${ }^{24}$

$$
\begin{aligned}
\operatorname{EEI}\left(\mu_{\mathrm{R}}\right) & =\frac{2}{3} i \pi^{2} \not k_{1} \int\left[d^{4} q\right] \frac{1}{\bar{q}^{2}\left(\bar{q}^{2}+k_{1}^{2}+2\left(q \cdot k_{1}\right)\right)}=E E I_{b}+E E I_{a}\left(\mu_{\mathrm{R}}\right) \\
& =V_{0} \frac{4-n}{3} \not k_{1}\left\{\int \frac{d^{n} q}{\mu_{\mathrm{R}}^{-2 \epsilon}} \frac{1}{q^{2}\left(q^{2}+k_{1}^{2}+2\left(q \cdot k_{1}\right)\right)}-\left.\lim _{\mu \rightarrow 0} \int \frac{d^{n} q}{\mu_{\mathrm{R}}^{-2 \epsilon}} \frac{1}{\bar{q}^{4}}\right|_{\mu=\mu_{\mathrm{R}}}\right\}
\end{aligned}
$$

where the last representation is suitable to be used to prove eq. (4.27). A further example of computation of an $E E I$ via sub-prescription is given in appendix $\mathrm{C}$.

Finally, it is important to realize that EEIs also arise in the intermediate steps of the calculation of the QCD correlation functions without external fermions discussed in section 4.2. From eq. (4.22) and the universality ${ }^{25}$ of the coupling constant extracted from all of the three-point vertices in figure 1 we infer that one must find zero when summing up the $E E I$ s from all contributing diagrams. Indeed, we have explicitly checked that this happens in the case of the $N_{f}$ corrections to the gluon and ghost propagators.

\section{Results}

The main result of our calculation is the list of QCD renormalization constants in appendix A. When setting $(\lambda, \delta)=(1,0)$ the known $\overline{\mathrm{MS}}$ formulae [20-22] are recovered. The

\footnotetext{
${ }^{22}$ This is obtained by anticommuting until the $\hat{q}_{2}$ meet.

${ }^{23}$ By doing so, we reintroduce the correct one-loop behaviour leading to (2.4).

${ }^{24}$ See appendix D.

${ }^{25}$ See section 5 .
} 
choice $(\lambda, \delta)=(0,1)$ corresponds to $\mathrm{D}_{\mathrm{Reg}}^{\mathrm{FDR}}$, i.e. the renormalization scheme which reproduces the FDR QCD Green's functions up to two loops.

As required by the QCD S-T identity

$$
\frac{Z_{G G G}}{Z_{G G}}=\frac{Z_{G c c}}{Z_{c c}}=\frac{Z_{G \Psi \Psi}}{Z_{\Psi \Psi}}=\sqrt{Z_{\alpha_{S}}} \sqrt{Z_{G G}},
$$

we have verified that the three ratios

$$
\frac{1}{Z_{G G}}\left(\frac{Z_{G G G}}{Z_{G G}}\right)^{2}, \quad \frac{1}{Z_{G G}}\left(\frac{Z_{G c c}}{Z_{c c}}\right)^{2}, \quad \frac{1}{Z_{G G}}\left(\frac{Z_{G \Psi \Psi}}{Z_{\Psi \Psi}}\right)^{2},
$$

produce the same coupling constant renormalization, namely $Z_{\alpha_{S}}(\lambda, \delta)$ in eq. (A.9). In the case of $Z_{\alpha_{S}}(0,1)$ this provides a stringent test on the universality of FDR.

Using $m_{q} \neq 0$ in $G_{3}^{\ell}$ gives the quark mass renormalization constant in eq. (A.10). Again the correct $\overline{\mathrm{MS}}$ result [21] is reproduced with $\lambda=1$ and $\delta=0$. As an extra check, we have verified that any $m_{q}$ dependence drops in $Z_{\Psi \Psi}$, as should be.

Eq. (A.9) can be used to infer the coupling constant shift between FDR and $\overline{\mathrm{MS}}$

$$
\begin{aligned}
\frac{Z_{\alpha_{S}}(1,0)}{Z_{\alpha_{S}}(0,1)}=\frac{\alpha_{S}^{\mathrm{FDR}}}{\alpha_{S}^{\overline{\mathrm{MS}}}}= & 1+\left(\frac{\alpha_{S}^{\overline{\mathrm{MS}}}}{4 \pi}\right) \frac{N_{c}}{3}+\left(\frac{\alpha_{S}^{\overline{\mathrm{MS}}}}{4 \pi}\right)^{2}\left\{\frac{89}{18} N_{c}^{2}+8 N_{c}^{2} f\right. \\
& \left.+N_{f}\left[N_{c}-\frac{3}{2} C_{F}-f\left(\frac{2}{3} N_{c}+\frac{4}{3} C_{F}\right)\right]\right\} .
\end{aligned}
$$

In an analogous way eq. (A.10) produces the quark mass shift

$$
\begin{aligned}
\frac{m_{q}^{\mathrm{FDR}}}{m_{q}^{\overline{\mathrm{MS}}}}= & 1-C_{F}\left(\frac{\alpha_{S}^{\overline{\mathrm{MS}}}}{4 \pi}\right)+C_{F}\left(\frac{\alpha_{S}^{\overline{\mathrm{MS}}}}{4 \pi}\right)^{2}\left\{\frac{77}{24} N_{c}-\frac{5}{8} C_{F}+f\left(9 N_{c}+\frac{11}{3} C_{F}\right)\right. \\
& \left.+N_{f}\left(\frac{1}{4}-\frac{2}{3} f\right)\right\} .
\end{aligned}
$$

eqs. (5.3) and (5.4) provide the transition rules from IR finite QCD quantities computed in FDR and their analogue in $\overline{\mathrm{MS}}$.

Finally, it is well known that one can use the coupling constant shift between two schemes at a given order to relate the two beta functions at one order higher. In the following we sketch out the two-loop proof of this [11] and use it to derive the three-loop beta function in FDR. Let us consider two different renormalization schemes defined in terms of two coupling constants $\alpha_{A}$ and $\alpha_{B}$ related by a shift

$$
\frac{\alpha_{A}}{4 \pi}=\frac{\alpha_{B}}{4 \pi}\left[1+c_{1}\left(\frac{\alpha_{B}}{4 \pi}\right)+c_{2}\left(\frac{\alpha_{B}}{4 \pi}\right)^{2}+O\left(\alpha_{B}^{3}\right)\right] .
$$

The two beta functions

$$
\beta_{A, B}=\mu \frac{d}{d \mu} \frac{\alpha_{A, B}}{4 \pi}=\left(\frac{\alpha_{A, B}}{4 \pi}\right)^{2}\left[b_{0}^{A, B}+b_{1}^{A, B}\left(\frac{\alpha_{A, B}}{4 \pi}\right)+b_{2}^{A, B}\left(\frac{\alpha_{A, B}}{4 \pi}\right)^{2}+O\left(\alpha_{A, B}^{3}\right)\right]
$$


are linked by the chain rule

$$
\beta_{A}=\mu \frac{d}{d \mu} \frac{\alpha_{A}}{4 \pi}=\mu\left(\frac{d}{d \mu} \frac{\alpha_{B}}{4 \pi}\right) \frac{d \alpha_{A}}{d \alpha_{B}}=\beta_{B} \frac{d \alpha_{A}}{d \alpha_{B}} .
$$

Writing both sides in terms of the same $\alpha$ through eq. (5.5) we find the standard result of scheme independence up to two loops, i.e. $b_{0}^{A}=b_{0}^{B}$ and $b_{1}^{A}=b_{1}^{B}$ as well as an expression for the three-loop beta function in scheme $B$, using only two-loop information from scheme $A$

$$
b_{2}^{B}=b_{2}^{A}+c_{1} b_{1}^{A}+\left(c_{1}^{2}-c_{2}\right) b_{0}^{A} .
$$

To calculate the three-loop beta function in FDR we use the three-loop $\overline{\mathrm{MS}}$ beta function in [23] together with the values from from eq. (5.3). This gives

$$
\begin{aligned}
b_{2}^{\mathrm{FDR}}= & N_{c}^{3}\left(-\frac{3610}{27}-\frac{176}{3} f\right)+N_{f}^{2}\left(-\frac{40}{9} C_{F}-\frac{43}{27} N_{c}+f\left(-\frac{16}{9} C_{F}-\frac{8}{9} N_{c}\right)\right) \\
& +N_{f}\left(\frac{1331}{27} N_{c}^{2}+\frac{292}{9} N_{c} C_{F}-2 C_{F}^{2}+f\left(\frac{140}{9} N_{c}^{2}+\frac{88}{9} N_{c} C_{F}\right)\right) .
\end{aligned}
$$

\section{$6 \quad$ FDH without evanescent quantities}

Recently it has been observed that the naive extension to non-SUSY theories of the original formulation of the FDH method breaks unitarity [7]. This was seen as the standard renormalization program fails to remove all UV poles at high enough perturbative orders. During our work, it proved of interest to try and investigate this problem from our renormalization scheme perspective. Using the technology developed in the previous sections we can easily perform an analysis of the validity of the interpretation of FDH as a perturbative description of QFT.

We begin by setting up the problem as in eqs. (1.1). We first recall eq. (1.1a) for an arbitrarily renormalized, dimensionally regulated correlation function. Next, we equate it to correlation functions calculated in the $\overline{\mathrm{FDH}}$ scheme up to two loops, i.e.

$$
\begin{aligned}
G_{\text {renormalized }}^{\ell} & =G_{\text {bare }}^{\ell}+(\ell \text {-loop-CTs })+\ldots+(1 \text {-loop-CTs }), \\
G_{\text {renormalized }}^{1} & =\overline{\mathrm{MS}}\left\{G_{\text {bare }}^{1}\left(n_{s}=4\right)\right\}, \\
G_{\text {renormalized }}^{2} & =\overline{\mathrm{MS}}\left\{G_{\text {bare }}^{2}\left(n_{s}=4\right)+\left.(1 \text {-loop-CTs })\right|_{n_{s}=4}\right\} .
\end{aligned}
$$

The Green's functions in the r.h.s. of eqs. (6.1b) and (6.1c) are calculated in the $\overline{\mathrm{FDH}}$ scheme by setting $n_{s}=4$ as dictated ${ }^{26}$ and subtracting both poles and universal constants through the $\overline{\mathrm{MS}}\{x\}$ operation.

If $\overline{\mathrm{FDH}}$ amounts to a different renormalization scheme to $\overline{\mathrm{MS}}$, then the $\ell$-loop-CTs should satisfy the renormalizability condition (3.4), i.e. they should be proportional to the Born and local. We can proceed to calculate these at each order in a similar way to section 4 ,

\footnotetext{
${ }^{26}$ Here and in the following we explicitly write the values of $n_{s}$ to highlight if contributions are regulated in FDH $\left(n_{s}=4\right)$ or DReg $\left(n_{s}=n\right)$. In a fully general FDH scheme $n_{s}$ is an arbitrary parameter, but we restrict to the $n_{s}=4$ case.
} 
making use of information from the FDR vacuum part. At one loop, the calculation is simple and provides the exact same $Z^{1}$ as FDR, indicating that $\overline{\mathrm{FDH}}$ exhibits the same coupling shift from $\overline{\mathrm{MS}}$. At two loops the computation is more complicated but in general we can write the following expression for what should be the two-loop counterterms:

$$
\begin{aligned}
(2-\text { loop-CTs })=\left.\overline{\mathrm{MS}}\{G V+S V-E E I+C T\}\right|_{n_{s}=4} \\
-\left.[G V+S V-E E I+C T]\right|_{n_{s}=n}
\end{aligned}
$$

We now split $S V$ and $E E I$ as in eqs. (4.21) and (4.26). As $n_{s}=n$ in the second line, we can make use of eq. (4.27). A direct calculation of the first line gives instead a striking different result for $n_{s}=4$ : the $C T$ part cancels the $b$ piece of the sub-vacuum, but not the EEI. In summary

$$
\begin{gathered}
(2 \text {-loop-CTs })=\left.\overline{\mathrm{MS}}\left\{G V+S V_{a}^{\prime}-E E I_{a}-E E I_{b}\right\}\right|_{n_{s}=4} \\
-\left.\left[G V+S V_{a}^{\prime}-E E I_{a}\right]\right|_{n_{s}=n} .
\end{gathered}
$$

This is problematic for FDH as the $E E I_{b}$ term does not in general satisfy the renormalizability condition. That is, we see that for $E E I_{b} \neq 0, \overline{\mathrm{FDH}}$ cannot be interpreted as a perturbative description of QFT. Nevertheless, as we have seen previously, the $E E I_{b}$ contribution is zero for external gauge states and so the only expected problems come with external fermions, just as experienced in [7].

The form of equation (6.3) naturally suggests a new definition of FDH which satisfies the renormalizability condition. Let us consider changing the bare two-loop FDH correlation function in the following way ${ }^{27}$

$$
G_{\text {bare }}^{2}\left|n_{s}=4 \rightarrow G_{\text {bare }}^{2}\right| n_{s}=4+\left.E E I_{b}\right|_{n_{s}=4}
$$

This now allows us to write down a working FDH analogue of eq. (4.28)

$$
\begin{aligned}
Z^{2, F D H^{\prime}} G^{0}=\left.\overline{\mathrm{MS}}\left\{G V+S V_{a}^{\prime}-E E I_{a}\right\}\right|_{n_{s}=4} \\
-\left.\left[G V+S V_{a}^{\prime}-E E I_{a}\right]\right|_{n_{s}=n} .
\end{aligned}
$$

Here we call this modified definition $\mathrm{FDH}^{\prime}$. Its renormalization constants $Z^{\ell, \mathrm{FDH}^{\prime}}$ are given in appendix $\mathrm{A}$ and correspond to the case $(\lambda, \delta)=(0,0)$. From these we are able to calculate the analogous version of eq. (5.1) in this scheme, indicating that the QCD S-T identity is respected, even with external fermions. ${ }^{28}$ What's more, we verify that in all cases the coupling constant shift agrees with the literature value [11], i.e. $\mathrm{FDH}^{\prime}$ is equivalent to FDH when the latter scheme makes sense and provides consistent predictions in all the other cases. Together these results suggest that, at least off-shell, this definition does not face the renormalization difficulties of the original FDH formulation. Furthermore, as we newly have control over the fermion sector, we are able to calculate a mass renormalization

\footnotetext{
${ }^{27}$ It is important to understand that the change we make in equation (6.4) can be read directly from the diagrams without the computation of counterterms.

${ }^{28}$ This is the important new case as the $E E I \mathrm{~s}$ are zero for the correlation functions with external gauge states.
} 
constant, and thereby a mass shift between $\overline{\mathrm{MS}}$ and $\mathrm{FDH}^{\prime}$

$$
\frac{m_{q}^{\mathrm{FDH}^{\prime}}}{m_{q}^{\overline{\mathrm{MS}}}}=1-C_{F}\left(\frac{\alpha_{S}^{\overline{\mathrm{MS}}}}{4 \pi}\right)+C_{F}\left(\frac{\alpha_{S}^{\overline{\mathrm{MS}}}}{4 \pi}\right)^{2}\left\{\frac{29}{12} N_{c}-\frac{13}{2} C_{F}+\frac{1}{4} N_{f}\right\} .
$$

Our new definition offers a different perspective than the recently proposed approaches to the unitarity based difficulties of FDH. Roughly speaking, any solution requires a connection between internal and external states. In the approach of [12], evanescent operators are introduced, as in dimensional reduction, to make the external states behave like the internal ones, thereby introducing a series of evanescent couplings. Conversely, our approach based upon sub-integration consistency makes internal states behave like external ones, and so does not require the introduction of any new fields whilst maintaining $n_{s}=4$ spin degrees of freedom.

\section{Summary and outlook}

As the FDR UV subtraction is consistently encoded in the definition of a four-dimensional and finite loop integration, the FDR approach to QFT does not require the introduction of counterterms in the Lagrangian. In particular, an order-by-order renormalization is avoided: the $\ell^{\text {th }}$ perturbative order is computed by only looking at $\ell$-loop Feynman diagrams.

We have proven, up to two loops in $\mathrm{QCD}$, that FDR is equivalent to a particular renormalization scheme of Dimensional Regularization, dubbed $\mathrm{D}_{\mathrm{Reg}}^{\mathrm{FDR}}$, whose renormalization constants, extracted from two- and three-point vertices, obey the Slavnov-Taylor identities. $\mathrm{D}_{\mathrm{Reg}}^{\mathrm{FDR}}$ and $\overline{\mathrm{MS}}$ are related by shifts in $\alpha_{s}$ and $m_{q}$ that we have explicitly computed. The transition rules derived in this paper can be used to translate calculations of IR finite quantities from FDR to $\overline{\mathrm{MS}}$.

During our analysis, we have identified a bottom-up interpretation of the difficulties in defining FDH beyond one loop. FDR naturally suggests a consistent definition by automatically generating the finite pieces needed to restore renormalizability. These extra terms are computed without introducing $\epsilon$-scalars nor evanescent quantities in the Lagrangian and can be directly read off from two-loop diagrams. Including such FDR inspired terms defines a consistent renormalizable scheme that we have called $\mathrm{FDH}^{\prime}$.

In this paper we have concentrated our focus on IR finite quantities. The possibility of consistently using FDR to regulate final state soft/collinear divergences at one loop has been proven in ref. [15]. The study of FDR in the presence of NNLO IR singularities is left for future investigations.

\section{Acknowledgments}

This research was supported by the European Commission through contracts ERC-2011AdG No 291377 (LHCtheory) and PITN-GA-2012-316704 (HIGGSTOOLS). We also thank the project FPA2013-47836-C3-1-P. Our figures are prepared with Axodraw [24]. 


\begin{tabular}{|c|c|c|c|}
\hline & $\mathrm{D}_{\mathrm{Reg}}^{\mathrm{FDR}}$ & $\overline{\mathrm{MS}}$ & $\mathrm{FDH}^{\prime}$ \\
\hline$\lambda$ & 0 & 1 & 0 \\
\hline$\delta$ & 1 & 0 & 0 \\
\hline
\end{tabular}

Table 1. $\lambda$ and $\delta$ in $\mathrm{D}_{\mathrm{Reg}}^{\mathrm{FDR}}, \overline{\mathrm{MS}}$ and $\mathrm{FDH}^{\prime}$.

\section{A The renormalization constants of the QCD vertices}

In the following we list the renormalization constants of the QCD correlation functions of figure 1 up to two loops in the Feynman-'t Hooft gauge

$$
Z_{i}(\lambda, \delta)=1+\left(\frac{\alpha_{S}}{4 \pi}\right) Z_{i}^{1}(\lambda, \delta)+\left(\frac{\alpha_{S}}{4 \pi}\right)^{2} Z_{i}^{2}(\lambda, \delta) .
$$

The three renormalization schemes we are interested in are parametrized by the values of $\lambda$ and $\delta$ given in table $1 . N_{c}$ is the number of colours, $N_{f}$ the number of active fermions, $C_{F}=\frac{N_{c}^{2}-1}{2 N_{c}}$ and $\alpha_{S}=\alpha_{S}(\lambda, \delta)$ is the QCD coupling constant of each scheme. The constant

$$
f=\frac{i}{\sqrt{3}}\left(\operatorname{Li}_{2}\left(e^{i \frac{\pi}{3}}\right)-\operatorname{Li}_{2}\left(e^{-i \frac{\pi}{3}}\right)\right)=-1.17195361 \ldots
$$

originates from the evaluation of the two-loop global vacuum in DReg,

$$
\begin{aligned}
Z_{G G}= & Z_{1}(\lambda, \delta) \\
= & +\frac{\alpha_{S}}{4 \pi}\left\{\frac{1}{\epsilon}\left[\frac{5}{3} N_{c}-\frac{2}{3} N_{f}\right]+\frac{1}{3} N_{c}(1-\lambda)\right\} \\
& +\left(\frac{\alpha_{S}}{4 \pi}\right)^{2}\left\{\frac{1}{\epsilon^{2}}\left[-\frac{25}{12} N_{c}^{2}+\frac{5}{6} N_{c} N_{f}\right]\right. \\
& +\frac{1}{\epsilon}\left[\frac{65}{24} N_{c}^{2}+\frac{1}{6} \lambda N_{c}^{2}+N_{f}\left(-C_{F}-\frac{5}{4} N_{c}\right)\right] \\
& +(1-\lambda) \frac{15}{8} N_{c}^{2}+\delta\left(\frac{9}{16} N_{c}^{2}+\frac{7}{2} N_{c}^{2} f\right) \\
& \left.+N_{f}\left(-(1-\lambda) C_{F}+\delta\left(\frac{7}{8} N_{c}-\frac{1}{2} C_{F}+f\left(-\frac{4}{3} C_{F}-\frac{1}{3} N_{c}\right)\right)\right)\right\}, \\
Z_{c c}= & Z_{2}(\lambda, \delta) \\
= & +\frac{\alpha_{S}}{4 \pi} \frac{1}{\epsilon}\left[\frac{1}{2} N_{c}\right] \\
& +\left(\frac{\alpha_{S}}{4 \pi}\right)^{2}\left\{\frac{1}{\epsilon^{2}}\left[-N_{c}^{2}+\frac{1}{4} N_{c} N_{f}\right]\right. \\
& +\frac{1}{\epsilon}\left[\frac{37}{48} N_{c}^{2}+\frac{1}{4} \lambda N_{c}^{2}-\frac{5}{24} N_{c} N_{f}\right] \\
& \left.+(1-\lambda) \frac{11}{48} N_{c}^{2}+\delta\left(\frac{19}{32} N_{c}^{2}+\frac{3}{2} N_{c}^{2} f\right)+\delta N_{f}\left(\frac{1}{16} N_{c}-\frac{1}{6} N_{c} f\right)\right\}, \\
Z_{\Psi \Psi}= & Z_{3}(\lambda, \delta) \\
= & +\frac{\alpha_{S}}{4 \pi}\left\{\frac{1}{\epsilon}\left[-C_{F}\right]+C_{F}(1-\lambda)\right\}
\end{aligned}
$$




$$
\begin{aligned}
& +\left(\frac{\alpha_{S}}{4 \pi}\right)^{2}\left\{\frac{1}{\epsilon^{2}}\left[N_{c} C_{F}+\frac{1}{2} C_{F}^{2}\right]\right. \\
& +\frac{1}{\epsilon}\left[-\frac{1}{4} C_{F}^{2}-\frac{17}{4} N_{c} C_{F}+\lambda C_{F}^{2}+\frac{1}{2} N_{f} C_{F}\right] \\
& +(1-\lambda)\left(\frac{13}{4} N_{c} C_{F}-\frac{1}{2} C_{F}^{2}\right)+\delta\left(-\frac{1}{8} C_{F}^{2}-\frac{19}{8} N_{c} C_{F}+f\left(-\frac{1}{3} C_{F}^{2}-4 N_{c} C_{F}\right)\right) \\
& \left.+N_{f}\left(-(1-\lambda) \frac{3}{4} C_{F}+\delta\left(C_{F}+\frac{4}{3} C_{F} f\right)\right)\right\} \\
& Z_{G G G}=Z_{4}(\lambda, \delta) \\
& =1+\frac{\alpha_{S}}{4 \pi}\left\{\frac{1}{\epsilon}\left[\frac{2}{3} N_{c}-\frac{2}{3} N_{f}\right]+\frac{1}{3} N_{c}(1-\lambda)\right\} \\
& +\left(\frac{\alpha_{S}}{4 \pi}\right)^{2}\left\{\frac{1}{\epsilon^{2}}\left[-\frac{13}{8} N_{c}^{2}+\frac{5}{4} N_{c} N_{f}\right]\right. \\
& +\frac{1}{\epsilon}\left[\frac{59}{48} N_{c}^{2}+\frac{1}{4} \lambda N_{c}^{2}+N_{f}\left(-C_{F}-\frac{25}{24} N_{c}\right)\right] \\
& +(1-\lambda) \frac{79}{48} N_{c}^{2}+\delta\left(-\frac{13}{32} N_{c}^{2}+\frac{5}{4} N_{c}^{2} f\right) \\
& \left.+N_{f}\left(-(1-\lambda) C_{F}+\delta\left(\frac{13}{16} N_{c}-\frac{1}{2} C_{F}+f\left(-\frac{4}{3} C_{F}-\frac{1}{6} N_{c}\right)\right)\right)\right\}, \\
& Z_{G c c}=Z_{5}(\lambda, \delta) \\
& =1+\frac{\alpha_{S}}{4 \pi} \frac{1}{\epsilon}\left[-\frac{1}{2} N_{c}\right] \\
& +\left(\frac{\alpha_{S}}{4 \pi}\right)^{2}\left\{\frac{1}{\epsilon^{2}}\left[\frac{5}{8} N_{c}^{2}\right]+\frac{1}{\epsilon}\left[-\frac{3}{8} N_{c}^{2}\right]+\delta\left(-\frac{3}{8} N_{c}^{2}-\frac{3}{4} N_{c}^{2} f\right)\right\}, \\
& Z_{G \Psi \Psi}=Z_{6}(\lambda, \delta) \\
& =1+\frac{\alpha_{S}}{4 \pi}\left\{\frac{1}{\epsilon}\left[-C_{F}-N_{c}\right]+C_{F}(1-\lambda)\right\} \\
& +\left(\frac{\alpha_{S}}{4 \pi}\right)^{2}\left\{\frac{1}{\epsilon^{2}}\left[-1+\frac{25}{8} N_{c}^{2}+\frac{1}{2} C_{F}^{2}-\frac{1}{4} N_{c} N_{f}\right]\right. \\
& +\frac{1}{\epsilon}\left[\frac{21}{8}-\frac{1}{4} C_{F}^{2}-\frac{181}{48} N_{c}^{2}+\lambda\left(-\frac{1}{2}+\frac{1}{4} N_{c}^{2}+C_{F}^{2}\right)+N_{f}\left(\frac{5}{24} N_{c}+\frac{1}{2} C_{F}\right)\right] \\
& +(1-\lambda)\left(-\frac{13}{8}+\frac{67}{48} N_{c}^{2}-\frac{1}{2} C_{F}^{2}\right) \\
& +\delta\left(\frac{19}{16}-\frac{1}{8} C_{F}^{2}-\frac{69}{32} N_{c}^{2}+f\left(2-\frac{1}{3} C_{F}^{2}-\frac{17}{4} N_{c}^{2}\right)\right) \\
& \left.+N_{f}\left(-(1-\lambda) \frac{3}{4} C_{F}+\delta\left(C_{F}-\frac{1}{16} N_{c}+f\left(\frac{1}{6} N_{c}+\frac{4}{3} C_{F}\right)\right)\right)\right\} \text {. }
\end{aligned}
$$

The above results can be used to derive the renormalization constant of the QCD coupling through eqs. (5.2)

$$
Z_{\alpha_{S}}(\lambda, \delta)=1+\frac{\alpha_{S}}{4 \pi}\left\{\frac{1}{\epsilon}\left[-\frac{11}{3} N_{c}+\frac{2}{3} N_{f}\right]-\frac{1}{3} N_{c}(1-\lambda)\right\}
$$




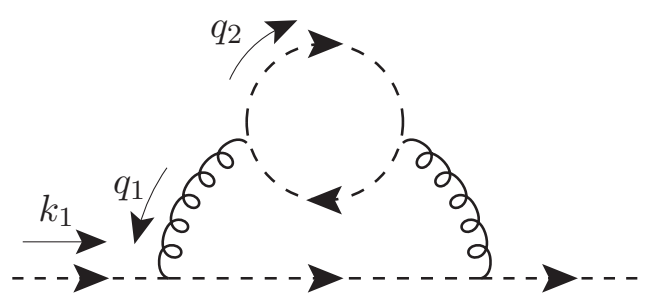

Figure 4. Two-loop diagram contributing to the ghost self-energy.

$$
\begin{aligned}
& +\left(\frac{\alpha_{S}}{4 \pi}\right)^{2}\left\{\frac{1}{\epsilon^{2}}\left[\frac{121}{9} N_{c}^{2}+\frac{4}{9} N_{f}^{2}-\frac{44}{9} N_{c} N_{f}\right]\right. \\
& +\frac{1}{\epsilon}\left[-\frac{29}{9} N_{c}^{2}-\frac{22}{9} \lambda N_{c}^{2}+N_{f}\left(\frac{11}{9} N_{c}+C_{F}+\frac{4}{9} \lambda N_{c}\right)\right] \\
& +\left(-(1-\lambda) \frac{20}{9} N_{c}^{2}+\delta\left(-\frac{5}{2} N_{c}^{2}-8 N_{c}^{2} f\right)\right) \\
& \left.+N_{f}\left((1-\lambda) C_{F}+\delta\left(\frac{1}{2} C_{F}-N_{c}+f\left(\frac{2}{3} N_{c}+\frac{4}{3} C_{F}\right)\right)\right)\right\} .
\end{aligned}
$$

Finally, computing $G_{\Psi \Psi}^{\ell}$ with $m_{q} \neq 0$ gives the renormalization constant associated with the quark mass

$$
\begin{aligned}
Z_{m_{q}}(\lambda, \delta)= & +\frac{\alpha_{S}}{4 \pi}\left\{\frac{1}{\epsilon}\left[-3 C_{F}\right]+C_{F}(1-\lambda)\right\} \\
& +\left(\frac{\alpha_{S}}{4 \pi}\right)^{2}\left\{\frac{1}{\epsilon^{2}}\left[\frac{11}{2} N_{c} C_{F}+\frac{9}{2} C_{F}^{2}-N_{f} C_{F}\right]\right. \\
& +\frac{1}{\epsilon}\left[-\frac{15}{4} C_{F}^{2}-\frac{85}{12} N_{c} C_{F}+\lambda\left(3 C_{F}^{2}-N_{c} C_{F}\right)+\frac{5}{6} N_{f} C_{F}\right] \\
& +(1-\lambda)\left(\frac{15}{2} C_{F}^{2}-\frac{11}{4} N_{c} C_{F}\right) \\
& -\delta\left(\frac{47}{8} C_{F}^{2}+\frac{19}{24} N_{c} C_{F}+f\left(\frac{11}{3} C_{F}^{2}+9 N_{c} C_{F}\right)\right) \\
& \left.+N_{f}\left(-(1-\lambda) \frac{1}{4} C_{F}+\delta \frac{2}{3} C_{F} f\right)\right\}
\end{aligned}
$$

\section{B A two-loop diagram}

As an example of the algorithm we use to extract and compute the FDR vacuum we work out in detail one among the two-loop diagrams contributing to $G_{2}^{2}$ in figure 1, namely the ghost-loop correction depicted in figure 4. The integrand of the corresponding amplitude is proportional to

$$
J\left(q_{1}, q_{2}\right)=\frac{\left(k_{1} \cdot q_{12}\right)\left(q_{1}+k_{1}\right) \cdot q_{2}}{q_{1}^{4} D_{1} q_{2}^{2} q_{12}^{2}},
$$

with $q_{12}=q_{1}+q_{2}$ and $D_{1}=\left(q_{1}+k_{1}\right)^{2}$. To read off $J\left(q_{1}, q_{2}, \mu^{2}\right)$ from $J\left(q_{1}, q_{2}\right)$ we apply the shift of eq. (2.2) in both the numerator and the denominator and simplify reducible 
numerators. This gives

$$
\begin{aligned}
J\left(q_{1}, q_{2}, \mu^{2}\right)= & \frac{\left(k_{1} \cdot q_{2}\right)^{2}}{\bar{q}_{1}^{4} \bar{D}_{1} \bar{q}_{2}^{2} \bar{q}_{12}^{2}}-\frac{k_{1}^{2}}{2} \frac{\left(k_{1} \cdot q_{2}\right)}{\bar{q}_{1}^{4} \bar{D}_{1} \bar{q}_{2}^{2} \bar{q}_{12}^{2}}+\frac{1}{2} \frac{\left(k_{1} \cdot q_{2}\right)}{\bar{q}_{1}^{4} \bar{q}_{2}^{2} \bar{q}_{12}^{2}}-\frac{k_{1}^{2}}{4} \frac{1}{\bar{q}_{1}^{4} \bar{D}_{1} \bar{q}_{2}^{2}}+\frac{1}{2} \frac{\left(k_{1} \cdot q_{2}\right)}{\bar{q}_{1}^{4} \bar{D}_{1} \bar{q}_{2}^{2}}+\frac{1}{4} \frac{1}{\bar{q}_{1}^{4} \bar{q}_{2}^{2}} \\
& +\frac{k_{1}^{2}}{4} \frac{1}{\bar{q}_{1}^{4} \bar{D}_{1} \bar{q}_{12}^{2}}-\frac{1}{2} \frac{\left(k_{1} \cdot q_{2}\right)}{\bar{q}_{1}^{4} \bar{D}_{1} \bar{q}_{12}^{2}}-\frac{1}{4} \frac{1}{\bar{q}_{1}^{4} \bar{q}_{12}^{2}}+\frac{k_{1}^{2}}{4} \frac{1}{\bar{q}_{1}^{2} \bar{D}_{1} \bar{q}_{2}^{2} \bar{q}_{12}^{2}}-\frac{\left(k_{1} \cdot q_{2}\right)}{\bar{q}_{1}^{2} \bar{D}_{1} \bar{q}_{2}^{2} \bar{q}_{12}^{2}} \\
& -\frac{1}{4} \frac{1}{\bar{q}_{1}^{2} \bar{q}_{2}^{2} \bar{q}_{12}^{2}}-\frac{1}{4} \frac{1}{\bar{q}_{1}^{2} \bar{D}_{1} \bar{q}_{2}^{2}}+\frac{1}{4} \frac{1}{\bar{q}_{1}^{2} \bar{D}_{1} \bar{q}_{12}^{2}}+\frac{1}{4} \frac{1}{\bar{q}_{2}^{2} \bar{D}_{1} \bar{q}_{12}^{2}} .
\end{aligned}
$$

Notice that the integrand manipulations we have performed so far are allowed both in FDR and DReg. In the following we concentrate on the first tensor

$$
J_{0}\left(q_{1}, q_{2}, \mu^{2}\right)=\frac{q_{2}^{\alpha} q_{2}^{\beta}}{\bar{q}_{1}^{4} \bar{D}_{1} \bar{q}_{2}^{2} \bar{q}_{12}^{2}},
$$

and explicitly derive the splitting

$$
J_{0}\left(q_{1}, q_{2}, \mu^{2}\right)=\left[J_{0, \mathrm{INF}}\left(q_{1}, q_{2}, \mu^{2}\right)\right]+J_{0, \mathrm{~F}}\left(q_{1}, q_{2}, \mu^{2}\right)
$$

needed to define the FDR integral

$$
\int\left[d^{4} q_{1}\right]\left[d^{4} q_{2}\right] J_{0}\left(q_{1}, q_{2}, \mu^{2}\right)=\lim _{\mu \rightarrow 0} \int d^{4} q_{1} d^{4} q_{2} J_{0, \mathrm{~F}}\left(q_{1}, q_{2}, \mu^{2}\right) .
$$

The other terms in eq. (B.1) are easier and can be treated analogously.

To analyze the UV behaviour of a two-loop integrand $J$ we introduce the four operators $d_{0}(J), d_{1}(J), d_{2}(J)$ and $d_{12}(J)$, which indicate how the integral over it behaves for large values of the integration momenta when $q_{1} \rightarrow \infty$ and $q_{2} \rightarrow \infty$ independently $\left(d_{0}(J)\right)$ or when $q_{i}$ is fixed $\left(d_{i}(J)\right)$. Thus, UV divergences occur when $d_{i}(J) \geq 0$ for some $i$. In our case, as

$$
d_{0}\left(J_{0}\right)=0, \quad d_{1}\left(J_{0}\right)=2, \quad d_{2}\left(J_{0}\right)<0, \quad d_{12}\left(J_{0}\right)<0,
$$

$J_{0}\left(q_{1}, q_{2}, \mu^{2}\right)$ has a logarithmic global UV divergence and is quadratically divergent in one of its sub-integrations. We now apply twice the identity

$$
\frac{1}{\bar{q}_{12}^{2}}=\frac{1}{\bar{q}_{2}^{2}}-\frac{q_{1}^{2}+2\left(q_{1} \cdot q_{2}\right)}{\bar{q}_{2}^{2} \bar{q}_{12}^{2}}
$$

and rewrite

$$
J_{0}\left(q_{1}, q_{2}, \mu^{2}\right)=\left[J_{1}\left(q_{1}, q_{2}, \mu^{2}\right)\right]-\left[J_{2}\left(q_{1}, q_{2}, \mu^{2}\right)\right]+J_{3}\left(q_{1}, q_{2}, \mu^{2}\right),
$$

with

$$
\begin{aligned}
& {\left[J_{1}\left(q_{1}, q_{2}, \mu^{2}\right)\right]=\frac{1}{\bar{q}_{1}^{4} \bar{D}_{1}}\left[\frac{q_{2}^{\alpha} q_{2}^{\beta}}{\bar{q}_{2}^{4}}\right],} \\
& {\left[J_{2}\left(q_{1}, q_{2}, \mu^{2}\right)\right]=\frac{q_{1}^{2}}{\bar{q}_{1}^{4} \bar{D}_{1}}\left[\frac{q_{2}^{\alpha} q_{2}^{\beta}}{\bar{q}_{2}^{6}}\right]+2 \frac{q_{1 \gamma}}{\bar{q}_{1}^{4} \bar{D}_{1}}\left[\frac{q_{2}^{\alpha} q_{2}^{\beta} q_{2}^{\gamma}}{\bar{q}_{2}^{6}}\right],}
\end{aligned}
$$




$$
J_{3}\left(q_{1}, q_{2}, \mu^{2}\right)=\frac{q_{2}^{\alpha} q_{2}^{\beta}\left(q_{1}^{2}+2\left(q_{1} \cdot q_{2}\right)\right)^{2}}{\bar{q}_{1}^{4} \bar{D}_{1} \bar{q}_{2}^{6} \bar{q}_{12}^{2}} .
$$

We see that $\left[J_{1}\left(q_{1}, q_{2}, \mu^{2}\right)\right]$ and $\left[J_{2}\left(q_{1}, q_{2}, \mu^{2}\right)\right]$ are factorizable integrands in which the $q_{2} \rightarrow \infty$ behaviour is fully parametrized in terms of divergent integrands depending only on $\mu^{2}$. Therefore, they belong to $\left[J_{0, \mathrm{INF}}\left(q_{1}, q_{2}, \mu^{2}\right)\right]$. In addition, since

$$
d_{0}\left(J_{3}\right)=0, \quad d_{1}\left(J_{3}\right)=0, \quad d_{2}\left(J_{3}\right)=0, \quad d_{12}\left(J_{3}\right)<0,
$$

further infinities need to be extracted from it, that is achieved by rewriting

$$
\frac{1}{\bar{D}_{1}}=\frac{1}{\bar{q}_{1}^{2}}-\frac{p^{2}+2\left(p \cdot q_{1}\right)}{\bar{q}_{1}^{2} \bar{D}_{1}}
$$

which gives

$$
J_{3}\left(q_{1}, q_{2}, \mu^{2}\right)=\left[J_{4}\left(q_{1}, q_{2}, \mu^{2}\right)\right]-J_{5}\left(q_{1}, q_{2}, \mu^{2}\right)-J_{6}\left(q_{1}, q_{2}, \mu^{2}\right)
$$

where

$$
\begin{aligned}
{\left[J_{4}\left(q_{1}, q_{2}, \mu^{2}\right)\right] } & =\left[\frac{q_{2}^{\alpha} q_{2}^{\beta}\left(q_{1}^{2}+2\left(q_{1} \cdot q_{2}\right)\right)^{2}}{\bar{q}_{1}^{6} \bar{q}_{2}^{6} \bar{q}_{12}^{2}}\right] \\
J_{5}\left(q_{1}, q_{2}, \mu^{2}\right) & =4 \frac{q_{2}^{\alpha} q_{2}^{\beta}\left(q_{1} \cdot q_{2}\right)^{2}\left(p^{2}+2\left(p \cdot q_{1}\right)\right)}{\bar{q}_{1}^{6} \bar{D}_{1} \bar{q}_{2}^{6} \bar{q}_{12}^{2}} \\
J_{6}\left(q_{1}, q_{2}, \mu^{2}\right) & =\frac{q_{2}^{\alpha} q_{2}^{\beta}\left(q_{1}^{4}+4 q_{1}^{2}\left(q_{1} \cdot q_{2}\right)\right)\left(p^{2}+2\left(p \cdot q_{1}\right)\right)}{\bar{q}_{1}^{6} \bar{D}_{1} \bar{q}_{2}^{6} \bar{q}_{12}^{2}} .
\end{aligned}
$$

Thus, the non-factorizable integrand $\left[J_{4}\left(q_{1}, q_{2}, \mu^{2}\right)\right]$ contributes to $\left[J_{0, \mathrm{INF}}\left(q_{1}, q_{2}, \mu^{2}\right)\right]$ and $J_{6}\left(q_{1}, q_{2}, \mu^{2}\right)$ is UV convergent. Furthermore, since

$$
d_{0}\left(J_{5}\right)<0, \quad d_{1}\left(J_{5}\right)=0, \quad d_{2}\left(J_{5}\right)<0, \quad d_{12}\left(J_{5}\right)<0,
$$

a logarithmic sub-divergence is still present in $J_{5}\left(q_{1}, q_{2}, \mu^{2}\right)$, that gets separated when applying once more eq. (B.6)

$$
J_{5}\left(q_{1}, q_{2}, \mu^{2}\right)=\left[J_{7}\left(q_{1}, q_{2}, \mu^{2}\right)\right]-J_{8}\left(q_{1}, q_{2}, \mu^{2}\right),
$$

with

$$
\begin{aligned}
{\left[J_{7}\left(q_{1}, q_{2}, \mu^{2}\right)\right] } & =4 \frac{q_{1 \gamma} q_{1 \delta}\left(p^{2}+2\left(p \cdot q_{1}\right)\right)}{\bar{q}_{1}^{6} \bar{D}_{1}}\left[\frac{q_{2}^{\alpha} q_{2}^{\beta} q_{2}^{\gamma} q_{2}^{\delta}}{\bar{q}_{2}^{8}}\right] \\
J_{8}\left(q_{1}, q_{2}, \mu^{2}\right) & =4 \frac{q_{2}^{\alpha} q_{2}^{\beta}\left(q_{1} \cdot q_{2}\right)^{2}\left(p^{2}+2\left(p \cdot q_{1}\right)\right)\left(q_{1}^{2}+2\left(q_{1} \cdot q_{2}\right)\right)}{\bar{q}_{1}^{6} \bar{D}_{1} \bar{q}_{2}^{8} \bar{q}_{12}^{2}} .
\end{aligned}
$$

In summary, $J_{0}\left(q_{1}, q_{2}, \mu^{2}\right)$ should be split as follows:

$$
\begin{aligned}
J_{0, \mathrm{~F}}\left(q_{1}, q_{2}, \mu^{2}\right) & =J_{8}\left(q_{1}, q_{2}, \mu^{2}\right)-J_{6}\left(q_{1}, q_{2}, \mu^{2}\right), \\
{\left[J_{0, \mathrm{INF}}\left(q_{1}, q_{2}, \mu^{2}\right)\right] } & =\left[J_{4}\left(q_{1}, q_{2}, \mu^{2}\right)\right]+\left[J_{1}\left(q_{1}, q_{2}, \mu^{2}\right)\right]-\left[J_{2}\left(q_{1}, q_{2}, \mu^{2}\right)\right]-\left[J_{7}\left(q_{1}, q_{2}, \mu^{2}\right)\right] .
\end{aligned}
$$

In the rest of the appendix we analyze the four terms contributing to $\left[J_{0, \mathrm{INF}}\left(q_{1}, q_{2}, \mu^{2}\right)\right]$ to establish their connection with $G V_{2}(\mu)$ and $S V_{2}(\mu)$ in eqs. (4.12)-(4.17). 
- $\left[J_{4}\left(q_{1}, q_{2}, \mu^{2}\right)\right]$ :

it gives a contribution to $G V_{2}(\mu)$. By using integration-by-parts one finds, for any value of $n$,

$$
\begin{aligned}
& \lim _{\mu \rightarrow 0} \int \frac{d^{n} q_{1}}{\mu_{\mathrm{R}}^{-2 \epsilon}} \frac{d^{n} q_{2}}{\mu_{\mathrm{R}}^{-2 \epsilon}}\left[J_{4}\left(q_{1}, q_{2}, \mu^{2}\right)\right] \\
& =g^{\alpha \beta} \lim _{\mu \rightarrow 0} \int \frac{d^{n} q_{1}}{\mu_{\mathrm{R}}^{-2 \epsilon}} \frac{d^{n} q_{2}}{\mu_{\mathrm{R}}^{-2 \epsilon}}\left\{\left(\frac{4}{3 n}-\frac{1}{4}\right)\left[\frac{1}{\bar{q}_{1}^{4} \bar{q}_{2}^{4}}\right]+\left(\frac{1}{6}-\frac{4}{3 n}\right)\left[\frac{1}{\bar{q}_{1}^{4} \bar{q}_{2}^{2} \bar{q}_{12}^{2}}\right]\right\} .
\end{aligned}
$$

- $\left[J_{1}\left(q_{1}, q_{2}, \mu^{2}\right)\right]$ :

since it is proportional to $\mu^{2}$, it vanishes in the limit $\mu^{2} \rightarrow 0$ we are interested in.

- $\left[J_{2}\left(q_{1}, q_{2}, \mu^{2}\right)\right]$ :

the odd-rank tensor in the r.h.s. of eq. (B.9) gives zero upon integration. On the other hand $\mu^{2} \rightarrow 0$ is allowed in the $q_{1}$ integrand multiplying the rank-two tensor. Thus

$$
\begin{aligned}
& \lim _{\mu \rightarrow 0} \int \frac{d^{n} q_{1}}{\mu_{\mathrm{R}}^{-2 \epsilon}} \frac{d^{n} q_{2}}{\mu_{\mathrm{R}}^{-2 \epsilon}}\left[J_{2}\left(q_{1}, q_{2}, \mu^{2}\right)\right] \\
& \quad=\frac{g^{\alpha \beta}}{4} \lim _{\mu \rightarrow 0} \int \frac{d^{n} q_{1}}{\mu_{\mathrm{R}}^{-2 \epsilon}} \frac{d^{n} q_{2}}{\mu_{\mathrm{R}}^{-2 \epsilon}}\left[\frac{1}{\bar{q}_{2}^{4}}\right]\left\{\left(\frac{1}{q_{1}^{2} D_{1}}-\left[\frac{1}{\bar{q}_{1}^{4}}\right]\right)+\left[\frac{1}{\bar{q}_{1}^{4}}\right]\right\} .
\end{aligned}
$$

The first term in the curly bracket contributes to $S V_{2}(\mu)$ with $A_{2}^{\prime}=-A_{2}=g^{\alpha \beta} / 4$, while the second to $G V_{2}(\mu)$ with $F_{22}=0$ and $F_{21}=g^{\alpha \beta} / 4$.

- $\left[J_{7}\left(q_{1}, q_{2}, \mu^{2}\right)\right]$ :

the integrand depending on $q_{1}$ in eq. (B.17) behaves as $1 / q_{1}^{4}$ when $q_{1} \rightarrow 0$. To disentangle the term which develops a $\ln \mu^{2}$ we rewrite 29

$$
\frac{q_{1 \gamma} q_{1 \delta}\left(p^{2}+2\left(p \cdot q_{1}\right)\right)}{\bar{q}_{1}^{6} \bar{D}_{1}}=\frac{q_{1 \gamma} q_{1 \delta}}{\bar{q}_{1}^{6}}-\frac{q_{1 \gamma} q_{1 \delta}}{\bar{q}_{1}^{4} \bar{D}_{1}}=\frac{q_{1 \gamma} q_{1 \delta}}{\bar{q}_{1}^{6}}-\frac{q_{1 \gamma} q_{1 \delta}}{q_{1}^{4} D_{1}}
$$

which results in

$$
\left[J_{7}\left(q_{1}, q_{2}, \mu^{2}\right)\right]=4\left(\left[\frac{q_{1 \gamma} q_{1 \delta}}{\bar{q}_{1}^{6}}\right]-\frac{q_{1 \gamma} q_{1 \delta}}{q_{1}^{4} D_{1}}\right)\left[\frac{q_{2}^{\alpha} q_{2}^{\beta} q_{2}^{\gamma} q_{2}^{\delta}}{\bar{q}_{2}^{8}}\right] .
$$

Since the integral over $q_{1}$ is UV finite

$$
\lim _{\mu \rightarrow 0} \int \frac{d^{n} q_{1}}{\mu_{\mathrm{R}}^{-2 \epsilon}} \frac{d^{n} q_{2}}{\mu_{\mathrm{R}}^{-2 \epsilon}}\left[J_{7}\left(q_{1}, q_{2}, \mu^{2}\right)\right]
$$

only contributes to $S V_{2}(\mu)$. By using tensor reduction and integration-by-parts identities one finds $A_{2}^{\prime}=-\frac{1}{24} g^{\mu \nu}+\frac{n-4}{24} p^{\mu} p^{\nu}$ and $A_{2}=\frac{1}{24} g^{\mu \nu}$ for this term.

\footnotetext{
${ }^{29}$ As mentioned in section 4 , this separation is in general achieved by using the propagator identity in eq. (4.15).
} 


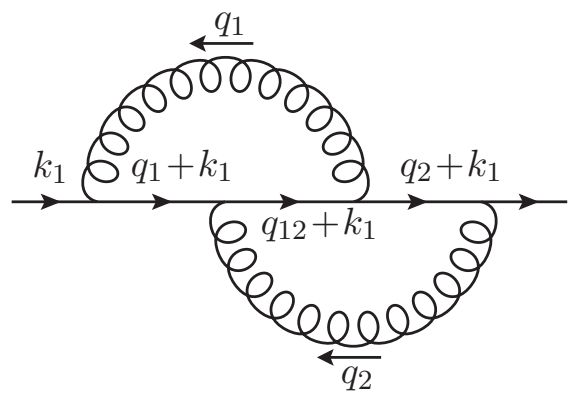

Figure 5. Diagram contributing to $G_{3}^{2}$. The fermion momenta follows the fermion line.

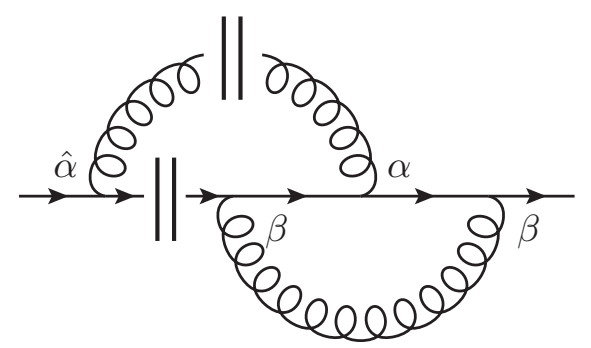

Figure 6. The same diagram of figure 5 from the point of view of the $q_{2}$ sub-integration.

\section{Sub-prescription example}

Here we discuss a further example of the sub-prescription in order to aid the understanding of the reader for future FDR calculations. Consider the FDR integral

$$
\int\left[\mathrm{d}^{4} q_{1}\right]\left[\mathrm{d}^{4} q_{2}\right] \frac{\gamma_{\beta}\left(\phi_{2}+k_{1}\right) \gamma_{\alpha}\left(q_{12}+k_{1}\right) \gamma^{\beta}\left(q_{1}+k_{1}\right) \gamma^{\alpha}}{\bar{q}_{1}^{2} \bar{q}_{2}^{2} \bar{D}_{1} \bar{D}_{2} \bar{D}_{12}},
$$

corresponding to the contribution to $G_{3}^{2}$ in figure 5 , where $\bar{D}_{i}=\bar{q}_{i}^{2}+k_{1}^{2}+2\left(q_{i} \cdot k_{1}\right)$. We wish to discuss how to extract the $E E I$ resulting from the sub-prescription, so as in figure 2 we consider the un-promoted numerator in order to find the relevant terms. In this diagram we have two sub-divergences, one for fixed $q_{1}$ and another fixed $q_{2}$. The terms from the sub-prescription of each can be extracted considering the sub-divergences independently. First we shall consider $q_{1}$ fixed. Let us disconnect the divergent sub-diagram, as in figure 6 . The numerator with its appropriate hatting reads

$$
N=\gamma_{\beta}\left(q_{2}+k_{1}\right) \gamma_{\alpha}\left(q_{12}+k_{1}\right) \gamma^{\beta}\left(q_{1}+k_{1}\right) \gamma^{\hat{\alpha}}
$$

and the numerator terms which give logarithmic divergences in $q_{2}$ are

$$
\begin{aligned}
N^{(2)} & =\gamma_{\beta} \phi_{2} \gamma_{\alpha} \phi_{2} \gamma^{\beta}\left(q_{1}+k_{1}\right) \gamma^{\hat{\alpha}} \\
& =-4 q_{2}^{2}\left(\phi_{1}+k_{1}\right)-4 \phi_{2}\left(\phi_{1}+k \phi_{1}\right) \not_{2} .
\end{aligned}
$$

The sub-prescription gives

$$
N^{(2)} \rightarrow N^{(2)}+\left.4 \mu^{2}\right|_{2}\left(\not q_{1}+k q_{1}\right),
$$


while the global prescription requires no change in $N^{(2)}$ :

$$
N^{(2)} \rightarrow N^{(2)}
$$

Thus, we find the resulting contribution by subtracting the (zero) global promotion and adding in the sub-promotion, leading to an $E E I$ of the form ${ }^{30}$

$$
E E I=4 \int\left[\mathrm{d}^{4} q_{1}\right]\left[\mathrm{d}^{4} q_{2}\right] \frac{\hat{\mu}_{2}^{2}\left(q_{1}+k k_{1}\right)}{\bar{q}_{1}^{2} \bar{q}_{2}^{2} \bar{D}_{1} \bar{D}_{2} \bar{D}_{12}}=i \pi^{2} \int\left[\mathrm{d}^{4} q_{1}\right] \frac{k_{1}}{\bar{q}_{1}^{2} \bar{D}_{1}} .
$$

When we move to perform the sub-prescription in the second sub-divergence, i.e. at fixed $q_{2}$, we make a similar treatment and find that the sub-prescription gives an identical contribution due to the symmetry of the diagram.

\section{Computing EEIs}

Given the important role played by the EEIs in the consistency of FDR we explicitly compute the extra-extra integrals in eqs. (4.36) and (C.6):

$$
\begin{aligned}
& E E I_{1}=\int\left[d^{4} q_{1}\right]\left[d^{4} q_{2}\right] \frac{\left.\hat{\mu}^{2}\right|_{2}\left(\phi_{1}+\not k_{1}\right)}{\bar{q}_{1}^{4} \bar{q}_{2}^{2} \bar{q}_{12}^{2}\left(\bar{q}_{1}^{2}+k_{1}^{2}+2 q_{1} \cdot k_{1}\right)} \\
& E E I_{2}=\int\left[\mathrm{d}^{4} q_{1}\right]\left[\mathrm{d}^{4} q_{2}\right] \frac{\hat{\mu}_{2}^{2}\left(\phi_{1}+k_{1}\right)}{\bar{q}_{1}^{2} \bar{q}_{2}^{2}\left(\bar{q}_{1}^{2}+k_{1}^{2}+2 q_{1} \cdot k_{1}\right)\left(\bar{q}_{2}^{2}+k_{1}^{2}+2 q_{2} \cdot k_{1}\right)\left(\bar{q}_{12}^{2}+k_{1}^{2}+2 q_{12} \cdot k_{1}\right)} .
\end{aligned}
$$

As a first step, we need the related one-loop extra (sub-)integrals

$$
I_{1}\left(p_{1}^{2}\right)=\int\left[d^{4} q\right] \frac{\mu^{2}}{\bar{q}^{2} \bar{D}_{1}^{2}}, \quad I_{2}=\int\left[d^{4} q\right] \frac{\mu^{2}}{\bar{q}^{2} \bar{D}_{1} \bar{D}_{2}},
$$

where $D_{i}=\left(q+p_{i}\right)^{2}$ and $\bar{D}_{i}=D_{i}-\mu^{2}$. To calculate them we start from the FDR defining expansions of their integrands with $\mu^{2} \rightarrow q^{2}$

$$
\begin{aligned}
\frac{q^{2}}{\bar{q}^{2} \bar{D}_{1}^{2}} & =\left[\frac{q^{2}}{\bar{q}^{4}}\right]-p_{1}^{2}\left[\frac{q^{2}}{\bar{q}^{6}}\right]-2\left[\frac{q^{2}\left(q \cdot p_{1}\right)}{\bar{q}^{6}}\right]+4\left[\frac{q^{2}\left(q \cdot p_{1}\right)^{2}}{\bar{q}^{8}}\right]+F_{1}(q), \\
\frac{q^{2}}{\bar{q}^{2} \bar{D}_{1} \bar{D}_{2}} & =\left[\frac{q^{2}}{\bar{q}^{6}}\right]+F_{2}(q),
\end{aligned}
$$

where $F_{1}(q)$ and $F_{2}(q)$ are UV convergent. $I_{1}\left(p_{1}^{2}\right)$ and $I_{2}$ are defined [6] as the difference between the l.h.s. of eq. (D.3) and the UV divergent part computed by changing back $q^{2} \rightarrow \mu^{2}$ in the numerator

$$
\begin{aligned}
I_{1}\left(p_{1}^{2}\right) & =\lim _{\mu \rightarrow 0} \mu^{2} \int d^{n} q\left(\frac{1}{\bar{q}^{2} \bar{D}_{1}^{2}}-\frac{1}{\bar{q}^{4}}+\frac{p_{1}^{2}}{\bar{q}^{6}}+2 \frac{\left(q \cdot p_{1}\right)}{\bar{q}^{6}}-4 \frac{\left(q \cdot p_{1}\right)^{2}}{\bar{q}^{8}}\right)=-i \pi^{2} \frac{p_{1}^{2}}{6}, \\
I_{2} & =\lim _{\mu \rightarrow 0} \mu^{2} \int d^{n} q\left(\frac{1}{\bar{q}^{2} \bar{D}_{1} \bar{D}_{2}}-\frac{1}{\bar{q}^{6}}\right)=\frac{i \pi^{2}}{2} .
\end{aligned}
$$

\footnotetext{
${ }^{30}$ See appendix D.
} 
Therefore we obtain

$$
\begin{aligned}
& E E I_{1}=\int\left[d^{4} q_{1}\right] \frac{\not q_{1}+\not k_{1}}{\bar{q}_{1}^{4}\left(\bar{q}_{1}^{2}+k_{1}^{2}+2 q_{1} \cdot k_{1}\right)} I_{1}\left(\bar{q}_{1}^{2}\right)=-\frac{i \pi^{2} \not k_{1}}{12} \int\left[d^{4} q_{1}\right] \frac{1}{\bar{q}_{1}^{2}\left(\bar{q}_{1}^{2}+k_{1}^{2}+2 q_{1} \cdot k_{1}\right)}, \\
& E E I_{2}=\int\left[\mathrm{d}^{4} q_{1}\right] \frac{\not q_{1}+k_{1}}{\bar{q}_{1}^{2}\left(\bar{q}_{1}^{2}+k_{1}^{2}+2 q_{1} \cdot k_{1}\right)} I_{2}=\frac{i \pi^{2} \not k_{1}}{4} \int\left[d^{4} q_{1}\right] \frac{1}{\bar{q}_{1}^{2}\left(\bar{q}_{1}^{2}+k_{1}^{2}+2 q_{1} \cdot k_{1}\right)} .
\end{aligned}
$$

Notice the replacement $I_{1}\left(q_{1}^{2}\right) \rightarrow I_{1}\left(\bar{q}_{1}^{2}\right)$ in accordance with the global prescription.

Finally, we point out the difference between hatting and not hatting $\left.\mu^{2}\right|_{2}$, i.e. the inequivalence between $E E I$ s and two-loop extra integrals. For instance

$$
E E I_{1}=-\frac{\pi^{4} \not k_{1}}{12}\left(\ln \frac{k_{1}^{2}}{\mu_{\mathrm{R}}^{2}}-2\right)
$$

while

$$
\int\left[d^{4} q_{1}\right]\left[d^{4} q_{2}\right] \frac{\left.\mu^{2}\right|_{2}\left(\not \not_{1}+\not k_{1}\right)}{\bar{q}_{1}^{4} \bar{q}_{2}^{2} \bar{q}_{12}^{2}\left(\bar{q}_{1}^{2}+k_{1}^{2}+2 q_{1} \cdot k_{1}\right)}=-\frac{\pi^{4} \not k_{1}}{12}\left(\ln \frac{k_{1}^{2}}{\mu_{\mathrm{R}}^{2}}+\frac{5}{3}+\frac{16}{3} f\right) .
$$

Open Access. This article is distributed under the terms of the Creative Commons Attribution License (CC-BY 4.0), which permits any use, distribution and reproduction in any medium, provided the original author(s) and source are credited.

\section{References}

[1] N. Bogoliubov and O. Parasiuk, On the multiplication of the causal function in the quantum theory of fields, Acta Math. 97 (1957) 227 [INSPIRE].

[2] K. Hepp, Proof of the Bogolyubov-Parasiuk theorem on renormalization, Commun. Math. Phys. 2 (1966) 301 [InSPIRE].

[3] W. Zimmermann, Convergence of Bogolyubov's method of renormalization in momentum space, Commun. Math. Phys. 15 (1969) 208 [InSPIRE].

[4] G. 't Hooft and M.J.G. Veltman, Regularization and renormalization of gauge fields, Nucl. Phys. B 44 (1972) 189 [inSPIRE].

[5] J.C. Collins, Renormalization, Cambridge University Press, Cambridge U.K. (1984).

[6] R. Pittau, A four-dimensional approach to quantum field theories, JHEP 11 (2012) 151 [arXiv: 1208.5457] [INSPIRE].

[7] W.B. Kilgore, Regularization schemes and higher order corrections, Phys. Rev. D 83 (2011) 114005 [arXiv: 1102.5353] [INSPIRE].

[8] R. Boughezal, K. Melnikov and F. Petriello, The four-dimensional helicity scheme and dimensional reconstruction, Phys. Rev. D 84 (2011) 034044 [arXiv:1106.5520] [INSPIRE].

[9] W.B. Kilgore, The four dimensional helicity scheme beyond one loop, Phys. Rev. D 86 (2012) 014019 [arXiv: 1205.4015] [inSPIRE].

[10] Z. Bern and D.A. Kosower, The computation of loop amplitudes in gauge theories, Nucl. Phys. B 379 (1992) 451 [INSPIRE]. 
[11] Z. Bern, A. De Freitas, L.J. Dixon and H.L. Wong, Supersymmetric regularization, two loop QCD amplitudes and coupling shifts, Phys. Rev. D 66 (2002) 085002 [hep-ph/0202271] [INSPIRE].

[12] A. Broggio, C. Gnendiger, A. Signer, D. Stöckinger and A. Visconti, Computation of $H \rightarrow g g$ in DRED and FDH: renormalization, operator mixing and explicit two-loop results, Eur. Phys. J. C 75 (2015) 418 [arXiv: 1503.09103] [INSPIRE].

[13] A.M. Donati and R. Pittau, Gauge invariance at work in FDR: $H \rightarrow \gamma \gamma$, JHEP 04 (2013) 167 [arXiv: 1302.5668] [INSPIRE].

[14] A.M. Donati and R. Pittau, FDR, an easier way to NNLO calculations: a two-loop case study, Eur. Phys. J. C 74 (2014) 2864 [arXiv:1311.3551] [INSPIRE].

[15] R. Pittau, QCD corrections to $H \rightarrow$ gg in FDR, Eur. Phys. J. C 74 (2014) 2686 [arXiv: 1307.0705] [INSPIRE].

[16] R. Pittau, On the predictivity of the non-renormalizable quantum field theories, Fortsch. Phys. 63 (2015) 132 [arXiv:1305.0419] [INSPIRE].

[17] R. Pittau, Integration-by-parts identities in FDR, Fortsch. Phys. 63 (2015) 601 [arXiv: 1408.5345] [INSPIRE].

[18] P. Nogueira, Automatic Feynman graph generation, J. Comput. Phys. 105 (1993) 279 [INSPIRE].

[19] J. Kuipers, T. Ueda, J.A.M. Vermaseren and J. Vollinga, FORM version 4.0, Comput. Phys. Commun. 184 (2013) 1453 [arXiv:1203.6543] [INSPIRE].

[20] E. Egorian and O.V. Tarasov, Two loop renormalization of the QCD in an arbitrary gauge, Teor. Mat. Fiz. 41 (1979) 26 [Theor. Math. Phys. 41 (1979) 863] [InSPIRE].

[21] T. Muta, Foundations of quantum chromodynamics: an introduction to perturbative methods in gauge theories, $3^{\text {rd }}$ ed., World Scientific, Singapore (2009).

[22] L.N. Mihaila, J. Salomon and M. Steinhauser, Renormalization constants and $\beta$-functions for the gauge couplings of the Standard Model to three-loop order, Phys. Rev. D 86 (2012) 096008 [arXiv: 1208.3357] [INSPIRE].

[23] S.A. Larin and J.A.M. Vermaseren, The three loop QCD $\beta$-function and anomalous dimensions, Phys. Lett. B 303 (1993) 334 [hep-ph/9302208] [INSPIRE].

[24] J.A.M. Vermaseren, Axodraw, Comput. Phys. Commun. 83 (1994) 45 [INSPIRE]. 